Research Articles: Systems/Circuits

\title{
Bi-directional communication between pontine nucleus incertus and the medial septum is carried out by electrophysiologically distinct neuronal populations
}

https://doi.org/10.1523/JNEUROSCI.0230-21.2022

Cite as: J. Neurosci 2022; 10.1523/JNEUROSCI.0230-21.2022

Received: 28 January 2021

Revised: 7 January 2022

Accepted: 10 January 2022

This Early Release article has been peer-reviewed and accepted, but has not been through the composition and copyediting processes. The final version may differ slightly in style or formatting and will contain links to any extended data.

Alerts: Sign up at www.jneurosci.org/alerts to receive customized email alerts when the fully formatted version of this article is published.

Copyright @ 2022 Trenk et al.

This is an open-access article distributed under the terms of the Creative Commons Attribution 4.0 International license, which permits unrestricted use, distribution and reproduction in any medium provided that the original work is properly attributed. 
Bi-directional communication between pontine nucleus incertus and the medial septum is carried out by electrophysiologically distinct neuronal populations

Abbreviated title:

Electrophysiological heterogeneity of NI neurons

Authors:

Aleksandra Trenk ${ }^{1}$, Magdalena Walczak ${ }^{1}$, Agata Szlaga $^{1}$, Kamil Pradel $^{1}$, Anna Blasiak $^{1}$, Tomasz Blasiak ${ }^{1}$

Affiliation:

${ }^{1}$ Department of Neurophysiology and Chronobiology, Institute of Zoology and Biomedical Research, Jagiellonian University, 9 Gronostajowa str., 30-387 Krakow, Poland

Corresponding authors' names and email address:

Aleksandra Trenk, aleksandra.trenk@uj.edu.pl; Tomasz Blasiak, tomasz.blasiak@uj.edu.pl

Number of:

- pages: 41;

- figures: 14,

- tables: 3.

Number of words for: - abstract: 248 ,

- introduction: 672, - discussion 1576.

Conflict of interest statement:

The authors declare no competing financial interests.

Acknowledgments:

This work has been supported by the Polish National Science Center grants: UMO2019/33/B/NZ4/03127 awarded to TB; UMO-2018/30/E/NZ4/00687 awarded to AB; doctoral scholarship "Etiuda VI" 2018/28/T/NZ4/00382 awarded to AT; doctoral scholarship "Etiuda VIII" 2020/36/T/NZ4/00341 and research grant "Preludium 14" 2017/27/N/NZ4/00785 awarded to KP, and the statutory funds of the Institute of Zoology and Biomedical Research. The open-access publication of this article was funded by the Priority Research Area BioS under the program "Excellence Initiative - Research University" at the Jagiellonian University in Krakow. 
Theta oscillations are one of the brain rhythms involved in memory formation, sensorimotor integration and control of locomotion and behavioural states. Generation and spatiotemporal synchronisation of theta oscillations rely on the interactions between nuclei forming a large neural network, part of which is pontine nucleus incertus (NI).

Here we identified distinct populations of NI neurons, based on the relationship of their firing to the hippocampal waves, with the special focus on theta oscillations, and the direction and type of interaction with the medial septum (MS) in male, urethane anaesthetised rats. By recording $\mathrm{NI}$ neuronal firing and hippocampal LFP we described NI neurons that fire action potentials in a theta phase-independent or theta phase-locked and delta wave-independent or delta wave-locked manner. Among hippocampal activity independent NI neurons, irregular, slow-firing and regular, fastfiring cells were observed, while hippocampal oscillations/waves locked $\mathrm{NI}$ neurons could be of bursting or non-bursting type. By projection specific opto-tagging, we revealed that only fast-firing theta phase-independent $\mathrm{NI}$ neurons innervate the MS, rarely receiving feedback information. In contrast, majority of theta bursting $\mathrm{NI}$ neurons were inhibited by MS stimulation and this effect was mediated by direct GABAergic input.

$$
\text { Described NI neuronal populations differ in reciprocal connections with the sept }
$$
hippocampal system, plausibly forming separate neuronal loops. Our results suggest that theta phase-independent $\mathrm{NI}$ neurons participate in theta rhythm generation through direct innervation of the MS, while theta-bursting NI neurons transmit further the rhythmic signal received from the MS to stabilise and/or strengthen rhythmic activity in other structures. 
The generation and spatiotemporal synchronisation of theta oscillations rely on the

51 interactions between nuclei forming a large neural network, part of which is the pontine nucleus

52 incertus $(\mathrm{NI})$. Here we describe that within $\mathrm{NI}$ there are populations of neurons that can be

53 distinguished based on the relationship of their firing to the hippocampal theta oscillations and delta

54 waves. We show that these neuronal populations largely do not have reciprocal connections with the

55 septohippocampal system, but form separate neuronal loops. Our results suggest that MS projecting,

56 fast-firing theta phase-independent NI neurons may participate in theta rhythm generation through

57 direct innervation of the MS, while theta-bursting $\mathrm{NI}$ neurons may transmit further the rhythmic

58 signal received from the MS to other structures.

59 


\section{Introduction}

Theta rhythm is one of the most abundant brain oscillatory phenomena, characterised by frequency in the $3-12 \mathrm{~Hz}$ band. This rhythm can be observed at the level of the electrical activity of neuronal populations (e.g. local field potential; Bland, 1986), in the rhythmic pattern of action potential firing of multiple or single neurons (e.g. rhythmic theta bursts; Bland et al., 2002; Di Prisco et al., 2002), and in the oscillations of neuronal membrane potential (Bland et al., 2002; Nunez et al., 1987; Strata, 1998). Theta rhythms present at various levels of the brain's organisation may interfere with each other and synchronise themselves in a large number of interconnected (directly and/or indirectly) structures (Buzsaki and Moser, 2013). Theta oscillations, spatio-temporally synchronised in nuclei forming functional networks, are a source of timing information used for memory coding and retrieval, sensorimotor integration, and processing of spatial information (Bland, 1986; Buzsaki, 1989; Buzsaki, 2002; Huerta and Lisman, 1993; Oddie and Bland, 1998; O'Keefe and Recce, 1993; Pavlides, et al. 1988; Vertes, 2005).

Several lines of evidence have shown that frequency and amplitude of hippocampal theta oscillations depend on the synergistic action of the bursting and tonic firing of theta-ON neurons located in the nuclei of the ascending brainstem hippocampal synchronising pathway. Tonically firing theta-ON neurons predominate at the brainstem levels of this system, while bursting theta-ON neurons are more likely to be found at the higher brain levels (Vertes et al., 2004). However, it has been shown that already at the level of brainstem and midbrain nuclei, bursting theta-ON neurons can be found, plausibly participating in the synchronisation and functional coupling of widely distributed structures responsible for different mnemonic functions (Di Prisco et al., 2002; Kocsis et al., 2001; Ma et al., 2013).

An important element of the ascending brainstem hippocampal synchronising pathway is nucleus incertus (NI; Brown and McKenna, 2015; Korotkova et al., 2018; Ma et al., 2018; Ryan et al., 2011). NI is a group of large, mostly GABAergic neurons located below the IV ventricle, expressing several markers, including relaxin-3 (RLN3), neuromedin B (NMB) and type 1 corticotropin-releasing 
factor receptor (Banerjee et al., 2010; Lu et al., 2020; Ma et al., 2013). NI neurons strongly innervate elements of the septohippocampal system of the mammalian brain (Goto et al., 2001; Olucha Bordonau et al., 2003; Teruel-Marti et al., 2008). In the rat medial septum, afferents from NI form dense plexus of symmetric (putatively inhibitory) terminals that synapse on the hippocampusprojecting neurons (Olucha Bordonau et al., 2012). Activation or inactivation of NI has been shown to induce or attenuate hippocampal theta rhythm, respectively (Nunez et al., 2006). Strong coherence between the $\mathrm{NI}$ local field potential and hippocampal theta has been described, as well as theta phase-locked firing of some NI neurons (Cervera Ferri et al., 2011; Ma et al., 2013). Interestingly, Martinez-Bellver et al. (2017) showed that there is a causal interaction between firing of NI neurons and the septohippocampal system. Recent studies have shown that there is great heterogeneity within the $\mathrm{NI}$, both at the functional, neurochemical and connectivity level. It was shown that the optogenetic activation of a subpopulation of NMB expressing $\mathrm{NI}$ neurons increases, whereas optogenetic inhibition of these cells decreases the power of hippocampal theta rhythm (Lu et al., 2020). On the other hand - as shown by another group - optogenetic activation of the GABAergic NI neurons, which also includes NMB-expressing cells decreased hippocampal theta power (Nasirova et al., 2020; Szonyi et al., 2019). These data show that the manipulation of different populations of NI neurons has different effects, both at the hippocampal theta and the behavioural levels. However, the electrophysiological identity of the NI neuronal subpopulations, their connections with the septohippocampal, and their relationship with hippocampal theta rhythm have not been well defined to date.

Therefore, the aim of our study was to identify $\mathrm{NI}$ neuronal populations that innervate and/or receive innervation from the medial septum and to determine the temporal relationship of their firing with the hippocampal theta oscillations and delta waves, which will better define neuronal circuits involved in hippocampal synchronisation. 


\section{Materials and methods}

\subsection{Experimental Design}

Animals and ethical approval. The experiments were performed on male Sprague-Dawley rats at the age of $8-12$ weeks and weight of $300-400 \mathrm{~g}$. The animals were bred at the Institute of Zoology and Biomedical Research Animal Facility at the Jagiellonian University in Krakow under standard conditions (12:12 light/dark cycle, light on at 08:00 h; temp. $20 \pm 2^{\circ} \mathrm{C}$; humidity $50 \pm 10 \%$ ) with food and water ad libitum. The experiments were approved by the $2^{\text {nd }}$ Local Institutional Animal Care and Use Committee (Krakow, Poland) and carried out in accordance with the EU Directive 2010/63/EU on the protection of animals used for scientific purposes. All efforts were made to minimise pain and discomfort, as well as the number of animals used in the procedures.

Viral vectors injections. The anaesthesia was induced by intraperitoneal (i.p.) injection of ketamine (100 mg/kg; Ketamina; Biowet, Poland) and xylazine (10 mg/kg; Sedazin; Biowet, Poland) mixture. To maintain anaesthesia, an additional $30 \%$ of the original dose of ketamine was injected during the procedure if needed. An anti-inflammatory drug was injected subcutaneously at the beginning of the surgery $(0.4 \%$ in saline; $0.1 \mathrm{ml} / 100 \mathrm{~g}$ animal body weight; Tolfedine; Vétoquinol; Biowet, Poland). After the disappearance of the corneal reflex and withdrawal response to paw pinch, the animal was mounted on the stereotaxic frame (SF-1450AP; ASI Instruments Inc., MI, USA) on the standard ear and incisor bars. During the whole procedure, the animal's body temperature was maintained at $37 \pm 0.5^{\circ} \mathrm{C}$ by an automatic heating pad (TCP-02; WMT, Poland). The eyes were protected from drying by the application of eye drops (Starazolin HydroBalance; Polpharma, Poland). After cutting the skin and exposing the bones of the skull vault, a trepanation hole was drilled to allow stereotactic access to the medial septum. A glass microcapillary tube (VITREX, Denmark) was processed on a vertical puller (PE-21; Narishige Scientific Instrument Lab., Japan) to obtain an ultrathin tip that was then broken to the final diameter of $40-50 \mu \mathrm{m}$. The injection needle prepared in this way was tightly connected to a Hamilton's syringe ( $1 \mu \mathrm{l}$; Hamilton; Bonaduz, Switzerland) and the whole system was filled with paraffin oil (Sigma-Aldrich, Poland). The tip of the microinjection 
capillary was backfilled with the viral vector just before it was lowered into the target structure. The medial septum of seventeen rats ( 8 for in vivo and 9 for ex vivo recordings; see below) was injected with an adeno-associated viral vector carrying genes for channelrhodopsin-2 (ChR2) and enhanced yellow fluorescent protein (EYFP), both expressed under the control of human synapsin (hSyn) promoter (AAV2-hSyn-hChR2(H134R)-EYFP; donated by Karl Deisseroth; UNC Vector Core, NC, USA; Zhang et al., 2006). The medial septum of fifteen rats was injected with a retrograde adenoassociated viral vector carrying genes for Chronos and green fluorescent protein (GFP), both expressed under the control of synapsin promoter (pAAV-Syn-Chronos-GFP; donated by Edward Boyden; Addgene viral prep \#59170-AAVrg; Addgene, MA, USA; Klapoetke et al., 2014). The following coordinates were used for medial septum injections: AP: $+0.6 \mathrm{~mm}, \mathrm{ML}: 0 \mathrm{~mm}, \mathrm{DV}:-7.2 \mathrm{~mm}\left(1^{\text {st }}\right.$ injection) and $-6.6 \mathrm{~mm}$ ( $2^{\text {nd }}$ injection) ventral from bregma (300 $\mathrm{nl}$ were injected at each ventral point). Finally, the scalp was sutured and the wound was covered with spray dressing (Nanosilver; Bioton, Poland) to promote healing. After surgery, the animals were allowed to recover for two weeks; their condition was monitored daily until the final experiment.

In vivo preparation. All in vivo electrophysiological experiments were performed on urethane anaesthetised rat preparation (urethane i.p. injection; $1.4 \mathrm{~g} / \mathrm{kg}$ diluted in saline; Sigma-Aldrich, Poland). The anaesthetised animal was mounted in the stereotaxic frame (SF-1450AP, ASI Instruments Inc.; Warren, MI, USA) on the standard ear and incisor bars. The tracheotomy was performed to reduce the mechanical instability of the brainstem resulting from the respiratory movements of the animal. During the whole experiment, the electrocardiogram (ECG) was monitored, and the body temperature was held at $37 \pm 0.5^{\circ} \mathrm{C}$ using an automatic heating pad (TCP02; WMT, Poland). After cutting the skin and exposing the bones of the skull vault, the bregma point was set below the level of the lambda point (usually $2.3 \mathrm{~mm}$ ) to obtain a rostral inclination of the skull of $15^{\circ}$, which allowed access to the nucleus incertus bypassing the confluence of superior sagittal sinus and transverse sinuses. Craniotomies were made above the areas of insertion of the electrodes and optical fibres, and after cleaning, the exposed brain surface was protected with a 
drop of paraffin oil (Sigma-Aldrich, Poland). The stereotactic coordinates of the examined brain structures were taken from the rat brain atlas (Paxinos and Watson, 2007), and then recalculated taking into account the rostral inclination of the skull.

Recording of the hippocampal local field potential. Local field potentials (LFP) were recorded with a blunt cut, Teflon coated stainless-steel wire (o.d.: $200 \mu \mathrm{m}$; Science Products, Germany) from stratum lacunosum moleculare of the hippocampal CA1 field (rostral angle 15, AP: $-4.6 \mathrm{~mm}, \mathrm{ML}: 3$ $\mathrm{mm}, \mathrm{DV}:-1.9 \mathrm{~mm}$ from the bregma). To verify the placement of the electrode, an electrolytic lesion (current of $0.6 \mathrm{~mA}$ applied for $1 \mathrm{~s}$ ) was made at the end of the recording. In the case of experiments with optogenetics, the LFP recording electrode was attached to the skull with dental cement (Duracryl Plus; SpofaDental, Czech Republic).

Multichannel in vivo recordings from the NI. The 32-channel multielectrode array (MEA; 4 shanks, 8 electrodes per shank; model A4x8-10mm-50-200-177; NeuroNexus, USA) was positioned in the nucleus incertus using the following stereotaxic coordinates: AP: $-9.2--9.7 \mathrm{~mm}, \mathrm{ML}: 0.1-$ $0.4 \mathrm{~mm}$, DV: $-7.5--7.2 \mathrm{~mm}$ from bregma (Paxinos and Watson, 2007). The spontaneous firing activity of $\mathrm{NI}$ neurons was recorded in one position in each animal. The extracellular signal picked up by MEA was digitised (40 kHz per channel), wide-band filtered $(0.77-7500 \mathrm{~Hz})$ and stored on a hard drive using OmniPlex D Neural Data Acquisition System (Plexon Inc., USA). To reconstruct the position of each MEA, its shanks were covered with a fluorescent dye (Dil; Thermo Fisher Scientific, USA) before brain implantation.

Optogenetic stimulation of the MS during the multichannel recording of $\mathrm{NI}$ electrical activity in vivo. Three weeks after the viral vector injection into the medial septum (AAV2-hSyn-hChR2(H134R)EYFP; as described above), the NI neurons' responses to medial septum optogenetic stimulation were observed. The LFP signal was recorded from the stratum lacunosum moleculare of the hippocampal CA1 field and electrical activity of NI neurons was extracellularly recorded with the multielectrode array, as described above. For the optogenetic stimulation, the optical fibre (core $\varnothing=100 \mu \mathrm{m}$, NA $=0.22$; Thorlabs, NJ, USA) was implanted so that its tip was aimed at the MS 
(coordinates of the tip: right angle $10^{\circ}, \mathrm{AP}: 0.7 \mathrm{~mm}, \mathrm{ML}: 0.7 \mathrm{~mm}$, DV: $-5.6 \mathrm{~mm}$ from the bregma; Paxinos and Watson, 2007). To verify the placement of the MEA and optical fibre, both were covered with a fluorescent dye (Dil; Thermo Fisher Scientific, USA) before brain implantation. The optical fibre was connected to a blue laser light source (wavelength: $473 \mathrm{~nm}$; model: MBL-III-473 with PSU-III-LED controller; CNI Optoelectronics Technology Co., Ltd., China). The light power never exceeded $10 \mathrm{~mW}$ as measured with the optical power meter (model: PM-100D equipped with S121C photodiode; ThorLabs, NJ, USA) at the tip of the optical fibre. Temporal parameters of the light pulses were digitally controlled by a micro1401 mk II laboratory interface and Spike2 software running a custom written script (Cambridge Electronic Design, UK). Protocols of optogenetic stimulation of MS included three frequencies from the theta band: $4 \mathrm{~Hz}$ ( 4 × $100 \mathrm{~ms}$ light pulses), $8 \mathrm{~Hz}$ ( 8 × $40 \mathrm{~ms}$ light pulses), $12 \mathrm{~Hz}$ (12 x 20 ms light pulses), one high-frequency pattern: $20 \mathrm{~Hz}$ (40 x $10 \mathrm{~ms}$ light pulse) and one single pulse (50ms) pattern. The given frequencies are based on intervals between light pulses' onsets. Stimulation at each frequency/pattern was applied at least 50 times at $10 \mathrm{~s}$ intervals.

Ex vivo recording: tissue preparation, data acquisition and optogenetic stimulation of medial septum originating terminals. Whole-cell patch clamp electrophysiological recordings were performed as previously described (Kania et al., 2020). Male Sprague-Dawley rats were deeply anesthetised with isoflurane (AErrane; Baxter, Poland) and decapitated between 02:00-03:00 ZT. The brains were collected in ice-cold, low-sodium, high-magnesium ACSF, containing (in mM): 185 sucrose, $25 \mathrm{NaHCO}_{3}, 3 \mathrm{KCl}, 1.2 \mathrm{NaH}_{2} \mathrm{PO}_{4}, 2 \mathrm{CaCl}_{2}, 10 \mathrm{MgSO}_{4}$ and 10 glucose $(\mathrm{pH}=7.4$; osmolality = $290-300 \mathrm{mOsmol} / \mathrm{kg}$ ) and cut into $250 \mu \mathrm{m}$ thick coronal sections on a vibrating microtome (VT 1000S; Leica Instruments, Germany). Sections containing the NI were transferred to an incubation chamber containing carbogenated, warm $\left(32^{\circ} \mathrm{C}\right) \mathrm{ACSF}$, containing (in $\mathrm{mM}$ ): $118 \mathrm{NaCl}, 25 \mathrm{NaHCO}_{3}, 3$ $\mathrm{KCl}, 1.2 \mathrm{NaH}_{2} \mathrm{PO}_{4}, 2 \mathrm{CaCl}_{2}, 1.3 \mathrm{MgSO}_{4}$ and 10 glucose $(\mathrm{pH}=7.4$; osmolality $290-300 \mathrm{mOsmol} / \mathrm{kg})$. After a recovery period $(90-120 \mathrm{~min})$, the slices were placed in a recording chamber, where the tissue was perfused $(2 \mathrm{ml} / \mathrm{min})$ with carbogenated, warm $\left(32^{\circ} \mathrm{C}\right)$ ACSF of the same composition as in the incubation chamber. Medial septum was also cut into $250 \mu \mathrm{m}$ thick coronal section on a vibrating 
microtome and viral vectors injection site was verified using Axio Imager M2 fluorescent microscope with an A-Plan 10×/0.25 objective. Recording micropipettes (7-9 $\mathrm{M} \Omega$; measured in the recording chamber filled with ACSF) were fabricated from borosilicate glass capillaries (BF100-78-10; Sutter Instruments, CA, USA) using a horizontal puller (P-97; Sutter Instruments, CA, USA) and filled with intrapipette solution containing (in $\mathrm{mM}$ ): 145 potassium gluconate, $2 \mathrm{MgCl}_{2}, 4 \mathrm{Na}_{2} \mathrm{ATP}, 0.4 \mathrm{Na}_{3} \mathrm{GTP}, 5$ EGTA, 10 HEPES (pH = 7.3; osmolality $290-300 \mathrm{mOsmol} / \mathrm{kg}$ ) and biocytin $(0.05 \%$, for subsequent immunofluorescent identification of recorded neurons). The calculated liquid junction potential was $+15 \mathrm{mV}$, and this value was subtracted from the recorded signal prior to the analysis. NI neurons were approached with a tip of the recording micropipette under visual control using an upright microscope (Examiner D1; Zeiss, Germany) equipped with video-enhanced infrared differential interference contrast. Cell-attached and subsequent whole-cell configurations were obtained using a negative pressure delivered by mouth suction. Voltage clamp recording and data acquisition were performed using an SEC 05LX amplifier (NPI, Germany) and Micro 1401 mk II (Cambridge Electronic Design, UK) converter operated by Signal and Spike2 software (Cambridge Electronic Design, UK) . Analogue signal filtered (low-pass filter: $3 \mathrm{kHz}$ ) and digitised $(20 \mathrm{kHz})$. All drugs used in the ex vivo experiments were applied via perfusion system. Voltage-clamp recordings (command potential -50 $\mathrm{mV}$ ) were performed in standard ACSF or ACSF containing gabazine (5 $\mu \mathrm{M}$; cat.no. 1262; Tocris, Poland) - a selective $\mathrm{GABA}_{\mathrm{A}}$ receptor antagonist. As the calculated reversal potential for $\mathrm{Cl}^{-}$currents for the solutions used in this study was $-90 \mathrm{mV}$, outward currents recorded at $-50 \mathrm{mV}$ holding potential were considered inhibitory (iPSCs, inhibitory postsynaptic currents), while inward currents were considered excitatory (ePSCs, excitatory postsynaptic currents). The tip of the optical fibre (core $\varnothing=200 \mu \mathrm{m}, \mathrm{NA}=0.5$; Thorlabs, NJ, USA) was positioned approximately $100 \mu \mathrm{m}$ above the surface of the brain slice, aiming at the tip of the recording micropipette positioned within the NI. The optical fibre was connected to a blue light emitting LED (wavelength: $465 \mathrm{~nm}$; PlexBright LED Module; Plexon Inc., USA). The power of the LED output was controlled by the current source (PlexBright LED Driver LD-1; Plexon Inc., TX, USA) and never exceeded $10 \mathrm{~mW}$, as measured with the optical power 
meter (PM-100D equipped with S121C photodiode; ThorLabs, NJ, USA) at the tip of the optical fibre. The temporal parameters of the light pulses were digitally controlled by a micro1401 mk II laboratory interface and Spike2 software running a custom written script. Optogenetic stimulation of MS originating terminals within the $\mathrm{NI}$ consisted of at least $20 \times 5 \mathrm{~ms}$ light pulses applied at $10 \mathrm{~s}$ interval. After the experiment brain slices were fixed and processed for histological examination as described below.

Optogenetic identification of NI neurons innervating the MS. Three weeks after the retrograde viral vector injection into the medial septum (pAAV-hSYN-Chronos-GFP; as described above), the NI neurons innervating the MS were optogenetically identified. The LFP signal was recorded from hippocampal CA1 field and electrical activity of $\mathrm{NI}$ neurons was extracellularly recorded with the multielectrode array, as described above. The optical fibre (core $\varnothing=200 \mu \mathrm{m}, \mathrm{NA}=0.5$; Thorlabs, NJ, USA) was implanted above the $\mathrm{NI}$ (coordinates of the tip: caudal angle $30^{\circ}, \mathrm{AP}:-9.6, \mathrm{ML}: 0 \mathrm{~mm}, \mathrm{DV}$ : 7.2 from bregma; Paxinos and Watson, 2007). The MEA and the optical fibre, before implantation, were covered with a fluorescent dye (Dil and DiD respectively; Thermo Fisher Scientific, USA) to allow subsequent verification of their location in the tissue. The optical fibre was connected to a blue light emitting LED (wavelength: 465 nm; model: PlexBright LED Module; Plexon Inc., USA). The power of the LED output was controlled by the precise current source (model: PlexBright LED Driver LD-1; Plexon Inc., USA) and never exceeded $12 \mathrm{~mW}$, as measured with the optical power meter (model: PM-100D equipped with S121C photodiode; ThorLabs, NJ, USA) at the tip of the optical fibre. Protocols of optogenetic stimulation of $\mathrm{NI}$ included eight patterns, each consisting of 50 light pulses: 5 ms pulses applied at frequencies: 10,30 and $50 \mathrm{~Hz}$ and $50 \mathrm{~ms}$ pulses applied at $1 \mathrm{~Hz}$. A neuron was considered to be directly driven by optogenetic stimulation (i.e., optogenetically tagged) if it responded with $100 \%$ fidelity to 10 and $30 \mathrm{~Hz}$ pulses, at least $90 \%$ fidelity to $50 \mathrm{~Hz}$ pulses, and responded with high-frequency discharges $(>50 \mathrm{~Hz})$ to laser light pulses of $50 \mathrm{~ms}$ duration.

Histological verification, immunohistochemical staining and image acquisition. At the end of each in vivo experiment, the animals were transcardially perfused with PBS followed by $4 \%$ formaldehyde 
solution. The extracted brains (in vivo experiments) or brain slices (ex vivo experiments) were kept overnight in $4 \%$ formaldehyde solution at $4{ }^{\circ} \mathrm{C}$. The brains were cut into $50 \mu \mathrm{m}$ coronal slices on the vibrating microtome (model: VT1000S; Leica, Germany) and the brain slices from the ex vivo experiments were processed histologically without further sectioning. To visualise biocytin filled neurons recorded ex vivo, fixed free-floating sections were blocked and permeabilised with $10 \%$ normal donkey serum (NDS) and 0.6\% Triton X-100 in PBS, respectively, at $4{ }^{\circ} \mathrm{C}$ overnight or for $3 \mathrm{~h}$ at room temperature. Subsequently, after washing in PBS, the sections were incubated with ExtrAvidin ${ }^{\circledR}-\mathrm{Cy}^{\mathrm{TM}}$ (1:200), 2\% NDS and 0.3\% Triton X-100 in PBS for 48 up to $72 \mathrm{~h}$ at $4{ }^{\circ} \mathrm{C}$ and, after several washing steps (in PBS), the slices were mounted on to glass slides and coverslipped with Fluoroshield with DAPI (Sigma-Aldrich, Poland). The brain sections from the in vivo experiments involving pAAV-Syn-Chronos-GFP injection to the MS (see above) were immunostained against GFP to improve the visibility of cells transfected with the viral vector. Sections were placed in $10 \%$ normalised donkey serum (NDS; Abcam, UK) and 0.6\% TritonX-100 (Sigma-Aldrich, Poland) in PBS for $1 \mathrm{~h}$ at room temperature to block the non-specific binding sites and permeabilisation, respectively. After washing in PBS, the slices were incubated for $24 \mathrm{~h}$ with rabbit anti-GFP antibodies (1:1000; Abcam, UK). Then, after another washing step, the sections were incubated overnight in the secondary donkey anti-rabbit AlexaFluor 488 antibody solution (1:400; Jackson ImmunoResearch, UK). After final rinsing, the sections were placed on slides and closed using Fluoroshield with DAPI (Sigma-Aldrich, Poland). The sections were imaged with a fluorescent microscope (Axio Imager.M2, camera: AxioCam MRm; objectives: A-Plan 10×/0.25 and EC-Plan-Neofluar 20x/0.25 Zeiss, Germany).

\subsection{Data processing and analysis}

All data were processed and analysed offline, except for the initial filtration of recorded signals as described above. The extracellular signal from the MEA recording spots located within the $\mathrm{NI}$ (as demonstrated by histological verification) was digitally filtered (high-pass filter: $300 \mathrm{~Hz}$ ) and subjected to spike detection in the Kilosort2 software (Pachitariu et al., 2016). The filtered signal 
and spike times of the sorted units were converted to the SON file format using a custom written MATLAB script (MathWorks, MA, USA), and then the quality of spike sorting was inspected and verified manually in Spike2 software (Cambridge Electronic Design, UK). The units that had percentage of refractory period violations ([inter-spike intervals $<2 \mathrm{~ms} /$ total number of intervals] *100) higher than $0.1 \%$, were rejected from further analysis. The final analysis of electrophysiological signal was carried out in NeuroExplorer and MATLAB software (NexTechnologies, CO, USA). Theta and delta epochs were identified based on percentage of spectral power exceeding $50 \%$ in theta $(3-$ $6 \mathrm{~Hz}$ ) and delta band $(0.1-3 \mathrm{~Hz})$, respectively, calculated for consecutive $4 \mathrm{~s}$ windows of the LFP signal recorded from the CA1 field (as described above). The full band $(0-500 \mathrm{~Hz})$ power spectral density was calculated with Fast Fourier Transform applied to the LFP signal with Welch's method (4 s windows). Zero phases (i.e., peak negativity) of theta and delta waves were detected with NeuroExplorer software algorithm (NexTechnologies, CO, USA) based on the method described elsewhere (Klausberger et al., 2003). Using the custom MATLAB script, each spike generated by the $\mathrm{NI}$ neuron was assigned a theta oscillation phase determined using the Hilbert transform. Based on the obtained values, the activity of each neuron was subjected to circular statistics using the MATALB CircStat toolbox (Berens, 2009) to determine whether, and if so, at which phase of hippocampal theta oscillation the given neuron preferentially generates action potentials. It was assumed that the firing of a neuron is theta phase-independent if the probability of generating action potential was uniformly distributed around the hippocampal theta cycle ( $p_{R}>0.05$, Rayleigh's test for uniformity) or direction vector length $r<0.2$ (at $p_{R} \leq 0.05$ ). If the probability was non-uniformly distributed around the hippocampal theta cycle $\left(p_{R} \leq 0.05\right)$ and the length of the direction vector was $\geq 0.2$, the neuron was identified as theta phase-locked (Lasztóczi et al., 2011). Theta phase preference of specific NI neuron populations was determined and compared using the Watson-Williams test (Watson and Williams, 1956). To determine whether the neuronal activity was locked with delta waves the peri-delta wave zero phase Z-score histograms (10ms bins) were generated using NeuroExplorer. The obtained histograms were analysed using custom-made MATLAB script. To each 
histogram a $5^{\text {th }}$ degree polynomial was fitted and the times and amplitudes of minimum and maximum closest to zero-phase were extracted. When at least one peak exceeded z-score value of 2.575 (99\% confidence level) the neuronal activity was considered correlated with the delta wave.

For each $\mathrm{NI}$ neuron, the median firing rate in theta and delta epochs was determined. The firing pattern of the recorded neuron (regular, irregular, bursting and non-bursting) was determined based on interspike interval and autocorrelation histograms generated for theta epochs. Bursts of action potentials and the parameters describing them were detected using a custom MATLAB script based on MLIB toolbox (Stüttgen, 2020). For each neuron, in each subsequent theta epoch, the spike density function (SDF; gaussian kernel, width $=25 \mathrm{~ms}$ ) and its mean value were calculated. The beginning of the burst was set on the first spike after the SDF exceeded the mean value, and the end of the burst was set on the last spike before the SDF returned below the mean value. The following parameters describing theta bursts and intensity of bursting were determined for each NI bursting neuron: length of the burst, number of spikes in the burst, intraburst frequency, bursting rate (i.e., bursts fired in time unit), \% of spikes generated in the bursts (i.e., [no of spikes in bursts/total number of spikes] *100\%), interburst interval and coefficient of variation of the interburst intervals.

To determine if neuron firing was correlated with each other, cross-correlograms (10ms bin, \pm 0.25 s delay) of activity in pairs of simultaneously recorded neurons using the NeuroExplorer were generated. The obtained cross-correlograms were analysed using custom-made MATLAB script. A seventh-degree polynomial was fitted to each cross-correlogram and the time and amplitude of the maximum closest to the zero lag were extracted, as well as the minimum preceding it. When at least one peak exceeded z-score value of 2.575 (99\% confidence level) the neuronal activity of the pair was considered correlated with one another.

To check whether the optogenetic stimulation of the MS elicited the reactions of NI neurons in vivo, the peristimulus spike density function (Gaussian kernel, width $=50 \mathrm{~ms}$ ) histograms were prepared using custom- Spike2 software (Cambridge Electronic Design, UK). Then, using custommade MATLAB scripts, the signal was divided into episodes of activity either above or below mean 
firing rate in the baseline, and next, their lengths and mean firing rates were extracted. Following, a multivariate Gaussian distribution was fitted to both parameters of baseline episodes and anomaly detection for the post-baseline episodes was performed. As a criterium for the detection the Mahalanobis distance was used; its value was set to 5.9915 which is equivalent to the Chi-square distribution-derived probability of 0.05 for two variables. The episodes lying outside of the distribution which latencies were shorter than $50 \mathrm{~ms}$ from the stimulation onset were classified as effects; the adjacent significant episodes were merged.

The amplitude of the light-evoked post synaptic currents recorded during the ex vivo experiments was determined based on the average response to 20 consecutive pulses (Spike2 software, Cambridge Electronic Design, UK). The distribution of the obtained parameters was tested for normality using the Shapiro-Wilk test. All values, because of their non-normal distribution, were presented as medians and ranged between the $1^{\text {st }}$ and $3^{\text {rd }}$ quartiles. Statistical evaluation of the differences between the medians was made using the Wilcoxon test (for paired data) or the MannWhitney test (for unpaired data) implemented in GraphPad Prism software (version 6; GraphPad Software, Inc., CA, USA). Differences with $p<0.05$ were considered statistically significant. 


\section{Results}

\subsection{Electrophysiological characterisation of NI neurons in vivo}

\subsubsection{Firing rate of the NI neurons during cortical activation and SWA}

The position of the microelectrode array in the nucleus incertus (NI) has been confirmed in 16 animals (Fig. 1A). The activity of $197 \mathrm{NI}$ neurons (no. of neurons/animal: average $=12$, range $=\langle 5,28>$ ) was recorded over at least one full, spontaneous cycle of brain state alternation, covering the phase of cortical activation and slow-wave activity (SWA). The firing rate of the majority of $\mathrm{NI}$ neurons $(87 \%, 171 / 197)$ was significantly higher during spontaneous cortical activation than during SWA state (theta-ON neurons; activation: median $=19.6 \mathrm{~Hz}, 1^{\text {st }} \& 3^{\text {rd }}$ quartile: $8.7 \& 38.7 \mathrm{~Hz}$; SWA: median $=9.8 \mathrm{~Hz}, 1^{\text {st }} \& 3^{\text {rd }}$ quartile: $4.0 \& 21.2 \mathrm{~Hz} ; \mathrm{n}=171, \mathrm{Z}=11.34, \mathrm{p}<0.0001$, Wilcoxon matched pairs test). The firing rate of the minority of $\mathrm{NI}$ neurons $(3 \%, 6 / 197)$ did not change across the brain states (theta unrelated; activation: median $=5.4 \mathrm{~Hz}, 1^{\text {st }} \& 3^{\text {rd }}$ quartile: $3.9 \& 26.9 \mathrm{~Hz}$; SWA: median $=3.9 \mathrm{~Hz}, 1^{\text {st }} \& 3^{\text {rd }}$ quartile: $2.4 \& 37.3 \mathrm{~Hz} ; \mathrm{n}=6, \mathrm{Z}=0.10, p=0.92$, Wilcoxon matched pairs test) or was significantly lower during the activation comparing to the SWA state (theta-OFF neurons; $10 \%, 20 / 197$; activation: median $=6.5 \mathrm{~Hz}, 1^{\text {st }} \& 3^{\text {rd }}$ quartile: 3.5 \& $16.6 \mathrm{~Hz}$; SWA: median $=$ 14.9 Hz, $1^{\text {st }} \& 3^{\text {rd }}$ quartile: $6.3 \& 22.2 \mathrm{~Hz} ; \mathrm{n}=20, \mathrm{Z}=2.80, \mathrm{p}<0.01$, Wilcoxon matched pairs test).

\subsubsection{Firing of $\mathrm{NI}$ neurons in relation to the phase of hippocampal theta oscillation}

Theta oscillations in the local field potential in the stratum lacunosum moleculare of the CA1 region of the hippocampus, observed during brain activation, were the basis for determining whether $\mathrm{NI}$ neurons generate action potentials in a theta phase-locked manner. $59 \%$ of recorded $\mathrm{NI}$ neurons (117/197) fired action potentials in theta phase-independent manner (Fig. 2A and 2C; theta phase-independent neurons; $p_{R}>0.05$, Rayleigh's test for uniformity or $p_{R} \leq 0.05$ and direction vector length $r<0.2)$, whereas firing of $41 \%$ of recorded NI neurons $(80 / 197)$ was significantly locked with the phase of the hippocampal theta oscillations (Fig. 2B and 2D; theta phase-locked neurons; $p_{R}<0.05$, Rayleigh's test for uniformity and direction vector length $r \geq 0.2$ ). Population of theta phase-locked NI neurons, observed in our study, had a significant preference to fire action potentials 
at the rising phase of the hippocampal theta oscillation recorded from the hippocampal CA1 field (mean direction $=134.0 \pm 7$ deg., direction vector length $\left.=0.55, p_{R}<<0.001, n=80\right)$. The phase preference of theta phase-locked NI neurons was not correlated either with their anteroposterior, lateromedial or dorsoventral position in the nucleus incertus $(r=0.09, p=0.35, r=0.33, p=0.07$ and $r=-0.09, p=0.44$ respectively; Zar, 1999).

\subsubsection{Firing patterns of the NI neurons during hippocampal theta oscillation}

In each group of $\mathrm{NI}$ neurons, both theta phase-independent and theta phase-locked, two additional subpopulations could be clearly differentiated, based on their pattern of electrical activity.

\section{Theta phase-independent NI neurons}

In the population of theta phase-independent $\mathrm{NI}$ neurons $(\mathrm{n}=117)$, the majority $(61 \%$, 71/117) were characterised by irregular, slow-firing of action potentials (Fig. 3A), whereas a minority (39\%, 46/117) were regular, fast-firing neurons (Fig. 3B). Regular, fast-firing NI neurons fired action potentials significantly faster and more regularly compared to the irregular, slow-firing neurons (Tab.1).

\section{Theta phase-locked NI neurons}

In the theta phase-locked group of $\mathrm{NI}$ neurons $(\mathrm{n}=80)$, the majority $(69 \%, 55 / 80)$ generated bursts of action potentials, as indicated by a bimodal distribution of interspike intervals and autocorrelation function plots with first and second order peaks (Fig. 3C). A smaller proportion of theta phase-locked $\mathrm{NI}$ neurons $(31 \%, 25 / 80)$ generated a non-bursting pattern of electrical activity (Fig. 3D). Bursting, theta phase-locked $\mathrm{NI}$ neurons fired action potentials significantly faster compared to the non-bursting, theta phase-locked neurons (Tab.1). The vast majority of theta phaselocked neurons was located in the nucleus incertus pars dissipata (Fig. 1C and 1D; laterality > 200 $\mu \mathrm{m})$, i.e., all bursting neurons $(100 \%, 55 / 55)$ and all but two non-bursting neurons $(92 \%, 23 / 25)$. Population of theta bursting NI neurons had a strong preference to fire action potentials at the rising phase of the hippocampal theta oscillation (Fig. 4A and 4B; mean direction $=126.0 \pm 6.8$ deg., 
direction vector length $=0.70, \mathrm{pR} \ll<0.001, \mathrm{n}=55$ ). On the other hand, non-bursting, theta phaselocked NI neurons, had uniformly distributed phase preferences and thus, this population of $\mathrm{NI}$ neurons shown no significant preference to fire action potentials at a particular phase of the hippocampal theta oscillation (Fig. 4A and 4C; mean direction $=169.8 \pm 16.8$ deg., direction vector length $=0.34, p R=0.051, n=25)$. The analysis of the correlation of parameters describing the strength of generated bursts and intensity of bursting with firing of $\mathrm{NI}$ theta bursting neurons was performed. It revealed that intraburst frequency and the number of spikes generated per burst were positively, linearly correlated with the firing rate of a neuron $\left(R^{2}=0.96\right.$ and $R^{2}=0.94$, respectively; Fig. $5 A$ and $5 B$ ), while the burst length did not show significant correlation (linear, $R^{2}=0.01$ or exponential, $\mathrm{R}^{2}=0.52$; Fig. $5 \mathrm{C}$ ). At the same time, bursting rate, interburst interval and $\mathrm{CV}$ of interburst interval were exponentially correlated with the firing rate (positive correlation, $R^{2}=0.82$ and negative correlations $R^{2}=0.94$ and $R^{2}=0.75$, respectively; Fig. $5 D, 5 E$ and $5 F$ ), while the percent of spikes generated in bursts did not show significant correlation (neither linear, R2 $=0.01$ nor exponential, $R^{2}=0.29$; Fig. $5 G$ ).

\subsubsection{Cross-correlation of NI neuronal firing during hippocampal theta oscillation}

A cross-correlation analysis of activity in pairs of simultaneously recorded neurons (during cortical activation), belonging to the same or different populations of $\mathrm{NI}$ neurons (as described above), was performed (Fig. 6). It revealed that firing of all theta bursting $\mathrm{NI}$ neurons is highly synchronised $(100 \%, 128 / 128$ pairs of neurons, peak z-score $>2.575$; median peak z-score $=39.8,1$ st \& 3rd quartile: $24.5 \&$ 56.6, median peak time $=0.02$ s, 1 st \& 3rd quartile: -0.01 \& 0.04; Fig. 6A). Similarly, majority of non-bursting, theta phase-locked neurons fired in synchrony with theta bursting ones $(95 \%, 98 / 103$ pairs of neurons, peak z-score $>2.575$; median peak $z$-score $=11.7$, 1 st $\& 3$ rd quartile: $7.5 \& 20.8$, median peak time $=0.04$ s, 1 st \& 3rd quartile: $0.01 \& 0.06 ;$ Fig. 7B). Only less than half of analysed pairs of non-bursting, theta phase-locked neurons was synchronised $(47 \%, 14 / 30$ pairs of neurons, peak z-score $>2.575$ ) what resulted in insignificant synchrony within this population of $\mathrm{NI}$ neurons (median peak z-score $=1.9,1$ st $\&$ 3rd quartile: $0.4 \& 3.3$, median peak time $=-0.02 \mathrm{~s}$, 
1st \& 3rd quartile: $-0.05 \& 0.02$; Fig. $6 C$ ). At the same time, a vast majority of analysed pairs of theta phase-independent $\mathrm{NI}$ neurons displayed asynchronous firing (cross-correlation peak z-score < 2.575): 93\% (127/136) of paired irregular firing neurons (Fig. 6D), 95\% (225/237) of paired regular \& irregular firing neurons (Fig. 6E) and 99\% (91/92) of paired regular firing neurons (Fig. 6F).

\subsubsection{Firing of $\mathrm{NI}$ neurons in relation to the delta waves during SWA}

Slow-wave activity observed in the local field potential in the CA1 region of the hippocampus was the basis for determining whether $\mathrm{NI}$ neurons generate action potentials in a delta wavedependent manner. Majority of recorded NI neurons (73\%; 144/197) fired action potentials independently of delta waves (Fig. 7A and 7C; delta wave-independent neurons; peak z-score of delta wave zero-phase triggered PSTH $<1.96)$, whereas firing of $27 \%$ of recorded $\mathrm{NI}$ neurons $(53 / 197)$ was significantly locked with the hippocampal delta waves (Fig. 7B and 7D; delta wave-locked neurons; peak z-score of delta wave zero-phase triggered PSTH peak $\geq 1.96)$. The latter population of neurons showed the highest probability of generating action potentials on the ascending phase of the delta wave, i.e., after its negative peak (median lag $=0.15 \mathrm{~s} ; 1^{\text {st }} \& 3^{\text {rd }}$ quartile: $0.09 \& 0.21 \mathrm{~s}$ ). Of the 144 delta wave-independent NI neurons, the majority $(69 \% ; 100 / 144)$ were neurons that, during cortical activation, were also theta phase-independent ( $85 \%$ of the theta phase-independent population; 100/117). The remaining 44 (31\%) delta wave-independent NI neurons, during cortical activation were theta phase-locked ( $55 \%$ of the theta phase-locked population; $44 / 80$ ). Of the 53 delta wavelocked $\mathrm{NI}$ neurons, the majority $(68 \% ; 36 / 53)$ were neurons that, during cortical activation, were theta phase-locked ( $45 \%$ of the theta phase-locked population; 36/80). The remaining 17 (32\%) delta wave-locked NI neurons, during cortical activation were theta phase-independent $(15 \%$ of the theta phase-independent population; 17/117). 


\subsubsection{Firing patterns of the NI neurons during hippocampal SWA}

In each group of $\mathrm{NI}$ neurons, both delta wave-independent and delta wave-locked, two

additional subpopulations could be clearly differentiated, based on their pattern of electrical activity.

\section{Delta wave-independent NI neurons}

In the population of delta wave-independent NI neurons $(n=144)$, the vast majority $(82 \%$, 118/144) were characterised by irregular, slow-firing of action potentials (Fig. 8A), whereas a minority $(18 \%, 26 / 118)$ were regular, fast-firing neurons (Fig. 8B). Regular, fast-firing NI neurons fired action potentials significantly faster and more regularly compared to the irregular, slow-firing neurons (Tab.2).

\section{Delta wave-locked NI neurons}

In the delta wave-locked group of $\mathrm{NI}$ neurons $(n=53)$, the majority $(62 \%, 33 / 53)$ generated a non-bursting pattern of electrical activity (Fig. 8C). A smaller proportion of delta wave-locked $\mathrm{NI}$ neurons $(38 \%, 20 / 53)$ generated bursts of action potentials, as indicated by a distribution of interspike intervals (dominant peak at short intervals and substantially smaller and slowly decaying count of longer intervals) and autocorrelation plot with first order peak sharply cantered around zero (Fig. 8D). Bursting, delta wave-locked NI neurons fired action potentials significantly faster compared to the non-bursting, delta wave-locked neurons (Tab. 2).

\subsubsection{Cross-correlation of NI neuronal firing during SWA}

A cross-correlation analysis of activity in pairs of simultaneously recorded neurons (during cortical SWA), belonging to the same or different populations of $\mathrm{NI}$ neurons (as described above), was performed (Fig. 9). It revealed that firing of all delta bursting $\mathrm{NI}$ neurons is highly synchronised $(100 \%, 19 / 19$ pairs of neurons, peak z-score $>2.575$; median peak z-score $=13.3,1$ st $\&$ 3rd quartile: $7.4 \& 17.0$, median peak time $=0.01 s, 1$ st $\&$ 3rd quartile: $-0.01 \& 0.02 ;$ Fig. 9A). Less than halve of non-bursting, delta wave-locked neurons fired in synchrony with delta bursting ones (44\%, 25/57 
pairs of neurons, peak z-score $>2.575$ ) what resulted in insignificant synchrony between these two populations of $\mathrm{NI}$ neurons (median peak z-score $=2.2$, 1st \& 3rd quartile: $1.0 \& 3.8$, median peak time $=-0.03 \mathrm{~s}, 1$ st \& 3rd quartile: $-0.09 \& 0.01 ;$ Fig. 9B). Similarly, only 39\% (16/41) of analysed pairs of non-bursting, delta wave-locked neurons were synchronised (peak z-score $>2.575$ ) what resulted in insignificant synchrony within this population of $\mathrm{NI}$ neurons (median peak $\mathrm{z}$-score $=-0.03,1$ st \& 3rd quartile: $-0.09 \& 0.01$, median peak time $=0.01$ s, 1 st \& 3rd quartile: $-0.04 \& 0.05 ;$ Fig. 9C). At the same time, a vast majority of analysed pairs of delta wave-independent NI neurons displayed asynchronous firing (cross-correlation peak z-score < 2.575): 78\% (459/588) of paired irregular firing neurons (Fig. 9D), 99\% (265/268) of paired regular \& irregular firing neurons (Fig. 9E) and all (29/29) of paired regular firing neurons (Fig. 9F).

\subsection{Nucleus incertus neurons' responses to input form the medial septum}

\subsubsection{Responses of the NI neurons to optogenetic stimulation of the medial septum in vivo}

The effective transfection of the medial septum (AAV2-hSyn-hChR2(H134R)-EYFP) as well as the position of the multielectrode array within the $\mathrm{NI}$ and the optical fibre above the MS was confirmed in four rats (Fig. 10A and 10B). The spontaneous activity of 63 nucleus incertus neurons (no. of neurons/animal: average $=16$, range $=\langle 7,28>$ ) and their responses to the optogenetic stimulation of the medial septum were recorded. Responses of 37 neurons were recorded during both, cortical activation and SWA, and the remaining neurons in only one of the cortical states. During cortical activation, majority $(86 \%, 6 / 7)$ of bursting theta phase-locked neurons were inhibited by the MS stimulation (Fig. 10C and 10E; Tab. 3) and cessation of each light pulse evoked inhibition was followed by rebound burst of action potentials. Theta phase-locked non-bursting neurons responded to MS activation with excitation and inhibition in equal proportions (40\%, 2/5; Fig. $10 \mathrm{C}$ and $10 \mathrm{E} ;$ Tab. 3). It is noteworthy that only a minority of theta bursting and non-bursting neurons (14\%, $1 / 7$ and $20 \%, 1 / 5$, respectively) did not respond to MS stimulation, and at the level of population, theta phase-locked NI neurons showed inhibitory response followed by rebound excitation (Fig. 10E). Interestingly, MS stimulation with a series of pulses at frequencies in the theta 
band $(4,8,12 \mathrm{~Hz})$ resulted in the synchronization of the theta bursting $\mathrm{NI}$ neurons to stimulation frequency (Fig. 11A). Conversely, high-frequency stimulation of the MS disrupted theta bursting of the $\mathrm{NI}$ neurons (Fig. $11 \mathrm{~B}$ and $11 \mathrm{C})$. On the other hand, $56 \%(9 / 16)$ and $44 \%(7 / 16)$ of theta phase independent, regular fast-firing neurons and irregular slow-firing neurons, respectively, did not responded to the MS optogenetic stimulation (Fig. 10C; Tab. 3). At the same time, excitatory and inhibitory responses were observed in a similar proportion in both theta-independent neuron populations (Fig. 10C; Tab. 3), resulting in no response at the population level (Fig. 10E). During cortical SWA, stimulation of MS inhibited majority of delta-bursting NI neurons, while among nonbursting delta wave-locked cells only excitatory responses were observed (Fig. 10D; Tab. 3). However, due to the small number of recorded neurons, it was not possible to conclusively determine whether, if at all, delta wave-locked neurons show a dominant response at the population level (Fig. 10F). In contrast, delta wave-independent neurons, although more frequently responded to MS stimulation with inhibition than excitation ( $25 \%$ vs. $15 \%$ and $44 \%$ vs. $11 \%$ respectively for irregular and regular firing neurons; Fig. 10D; Tab. 3), did not show a dominant response at the population level (Fig. 10F). Among NI neurons, whose responses to MS stimulation were observed in both cortical states $(n=37), 19 \%(7 / 32)$ were excited, $24 \%(9 / 37)$ were inhibited and 30\% (11/37) did not respond in both, cortical activation and SWA (Fig. 10G). Relatively small fraction of NI neurons responded to MS stimulation in only one cortical state, and none changed the direction of the response between cortical states (Fig. 10G).

\subsubsection{Responses of the NI neurons to optogenetic stimulation of the medial septum originating} terminals - ex vivo recordings

To investigate the functional properties of the MS innervation of the NI, whole-cell voltage clamp recordings were performed using brain slices from animals that were intra-MS injected with AAV2-hSyn-hChR2(H134R)-EYFP (Fig. 12A). In 15 out of 55 recorded NI neurons, optogenetic stimulation of MS originating fibres evoked inhibitory postsynaptic currents (IPSCs; Fig. 12B and 12C) and only one neuron responded with excitatory postsynaptic currents (EPSCS). IPSCs evoked by MS 
fibre stimulation had short latency $(2.5 \pm 0.3 \mathrm{~ms}, \mathrm{n}=15)$, they disappeared at chloride reversal potential $(-90 \mathrm{mV} ; 9 / 9$ neurons; Fig. $12 \mathrm{C})$, and were blocked by the selective $\mathrm{GABA}_{\mathrm{A}}$ receptor antagonist gabazine (5 $\mu \mathrm{M} ; 7 / 7$ neurons; Fig. 12D and 12E). No differences were detected in the proportions of inhibition/excitation and IPSCS/EPSCs evoked by MS axonal endings stimulation observed in in vivo and ex vivo recordings, respectively ( $p<0.05$; two-tailed, Fisher's exact test).

\subsection{Optogenetic identification of the NI neurons that innervate the medial septum}

In animals whose medial septum was transfected with the retrograde viral vector (pAAV-hSYN-Chronos-GFP; Fig. 13A), the direct responses of $22 \mathrm{NI}$ neurons to optogenetic stimulation were observed (Fig. 13B; 10 animals; opto-tagged $\mathrm{NI}$ neurons/animal: average $=2.2$, range $=\langle 1,5>$ ). All $\mathrm{NI}$ neurons identified as innervating medial septum belonged to the regular, fastfiring theta phase-independent type of cells (100\%, 22/22; Fig. 13D) that, during cortical SWA fired in delta wave-independent manner (59\%, 13/22 were irregular, slow firing; 21\%, 9/22 were regular slow firing).

\section{Discussion}

In the current study we identified two distinct NI neuronal populations, based on the relationship of their firing to the phase of the hippocampal theta rhythm and the direction and interaction with the medial septum: theta phase-independent and theta phase-locked neurons. Theta phase independent $\mathrm{NI}$ neurons included irregular, slow-firing and regular, fast firing cells. Theta phase locked neurons had a strong preference to fire action potentials at the rising phase of the hippocampal theta oscillation recorded from the stratum lacunosum moleculare of the hippocampal CA1 field (SLM). Interestingly, theta phase-locked NI neurons could be of bursting or non-bursting type. Theta-bursting NI neurons may have been omitted in previous studies due to the localisation of these cells mainly in the lateral parts of the NI (Ma et al., 2013). During the cortical SWA, based on the relation of action potential firing to hippocampal delta waves, we were able to distinguish two groups of NI neurons: delta wave-independent and delta wave-locked. Delta wave- 
independent $\mathrm{NI}$ neurons could be of irregular, slow-firing or regular, fast firing type and delta wavelocked neurons could be of bursting and non-bursting type. Delta wave-locked NI neurons fired action potentials preferentially on the ascending phase of the delta waves. In line with previous studies, for most of the $\mathrm{NI}$ neurons we noted an increased firing during cortical activation and a decreased discharge rate in the presence of slow-wave activity (Nunez et al., 2006; Martinez-Bellver et al., 2015). By projection specific opto-tagging, we determined that only fast firing theta phaseindependent $\mathrm{NI}$ neurons innervate the medial septum (MS). On the other hand, among neurons of this type, majority was unresponsive to optogenetic stimulation of the MS. In contrast, majority of theta bursting $\mathrm{NI}$ neurons were inhibited by the MS stimulation, and this effect was mediated by direct GABAergic input.

The knowledge gathered to date about nucleus incertus implies that it is a strong modulator of theta oscillations, however the involvement of $\mathrm{NI}$ in theta rhythm generation seems to be complex. Fibres originating from the NI heavily innervate the MS, and a recent study showed that GABAergic, including parvalbumin expressing, and glutamatergic MS neurons express mRNA for cognate relaxin-3 receptors, which makes these neurons a possible target of $\mathrm{NI}$ innervation (AlbertGasco et al., 2018; Haidar et al., 2019; Olucha-Bordonau et al., 2012). Importantly, MS parvalbumin GABAergic neurons were shown to rhythmically hyperpolarise hippocampal interneurons and promote theta oscillations (Hangya et al., 2009). The latest studies revealed that NMB expressing NI neurons project to the MS and are involved in the control of locomotion, arousal, and hippocampal theta oscillations (Lu et al., 2020; Nasirova et al., 2020). Given that half of the NMB neurons coexpress RLN3, it is not surprising that the relaxinergic input from the $\mathrm{NI}$ to the MS was also shown to be involved in theta rhythm modulation (Ma et al., 2009; Olucha-Bordonau et al., 2012; Ma et al., 2013; Haidar et al., 2017; Albert-Gasco et al., 2018; Szonyi et al., 2019). However, until now, the electrophysiological features of the NI neurons innervating the MS were unknown. Our results show that only regular, fast-firing theta phase-independent NI neurons project to the medial septum. In the light of the research by Szonyi et al. (2019), who showed that the NI neurons innervating MS give 
collaterals to the oriens-lacunosum-moleculare (OLM) neurons of the hippocampus, it can be assumed that the regular, fast-firing $\mathrm{NI}$ neurons identified by us can also innervate this population of hippocampal interneurons (Fig. 14).

Our data show that majority of the recorded regular, fast-firing theta phase-independent neurons innervating the MS were insensitive to the MS stimulation, and only the minor fractions responded with relatively weak excitation or inhibition. This indicates that the NI neurons innervating the MS do not receive strong MS feedback. At the same time, majority of theta-bursting neurons responded to the optogenetic stimulation of the MS with initial inhibition followed by a burst of action potentials. This rebound excitation was evident during rhythmic stimulation of the MS with frequencies across the theta band. Additionally, high-frequency stimulation eliminated spontaneous theta bursts, but still synchronised the NI neurons to the frequency of stimulation, which indicates strong control of theta-bursting NI neurons by the input from the MS. Based on these results, it is likely that the NI bursting neurons are innervated by one of the two identified GABAergic medial septal bursting neuron populations, one of which is coupled to the peak and the other to the trough of hippocampal theta-wave (observed in the SLM; Borhegyi et al., 2004; Fig. 14).

Theta bursting MS neurons, by direct innervation of the hippocampal interneurons, basket and OLM cells (Fig. 14), cause their rhythmic hyperpolarisations followed by rebound bursts. A similar mechanism may underlie the rhythmic activity of the $\mathrm{NI}$ bursting neurons that receive MS innervation, as described in our study. This is further supported by our observation that higher strength of bursts and intensity of bursting occurred in NI neurons having higher firing rates, while the proportion of action potentials generated in bursts and length of bursts were not correlated with the firing rate. Moreover, the temporal convergence of the bursts of the $\mathrm{NI}$ neurons and hippocampal basket cells allows to hypothesise that both these populations may be innervated by the same population of rhythmically bursting GABAergic MS neurons (Fig. 14). In this way, thetabursting $\mathrm{NI}$ neurons can use the pace received from the MS to efficiently participate in the synchronisation of oscillatory activity in the brain areas involved in locomotion or memory formation 
(Goto et al., 2001; Olucha-Bordonau et al., 2003). Directional information flow between the NI and the hippocampus and coherence between the field activity observed in both structures during theta oscillations suggest that the hippocampus is one of the target structures of $\mathrm{NI}$ theta-bursting cells (Cervera-Ferri et al., 2011; Martinez-Bellver et al., 2017).

Additionally, our in vivo recordings showed that a fraction of the non-bursting theta phaselocked and irregular, slow-firing theta phase-independent $\mathrm{NI}$ neurons responded to the MS stimulation, and their responses were heterogeneous (inhibition or excitation). At the same time, our ex vivo results revealed that $M S$ fibres optostimulation evoked inhibitory, $G_{A B} A_{A}$-dependent postsynaptic currents in a vast majority (all but one) of the recorded $\mathrm{NI}$ neurons. This suggests that the direct input from MS into NI is largely inhibitory. Concurrently, a recent study in mice has shown that stimulation of MS terminals within the $\mathrm{NI}$ elicited both GABA and glutamate dependent responses (Lu et al., 2020). These results indicate possible species differences in MS-NI innervation, as previously shown for some of the features of the NI originating RLN/RXFP3 system in mice and rats (Ma et al., 2017a).

Several studies have reported that NI activation or inhibition affects theta oscillations (Nunez et al., 2006; Martinez-Bellver et al., 2017; Ma et al., 2017b; Szonyi et al., 2019; Lu et al., 2020), but the results remain inconclusive. Some of the discrepancies may result from methodological differences, such as the used species (mouse/rat), state of the animal (anaesthetised/freely moving), stimulation method (electrical/optogenetic) or the group of activated cells (GABAergic/RLN3positive/NMB-positive). In one of the recent studies, all GABAergic NI neurons were optogenetically activated with light pulses applied at $25 \mathrm{~Hz}$ (Szonyi et al., 2019), which resulted in a decrease in the power of hippocampal theta oscillations. Such stimulation protocol - while inducing activity of regular, fast-firing theta phase-independent $\mathrm{NI}$ neurons that is close to their physiological rate and pattern of firing - at the same time can desynchronise rhythmic firing of the theta-bursting $\mathrm{NI}$ neurons. If, as we postulate from our observations, the function of theta bursting $\mathrm{NI}$ neurons is to enhance the synchronisation in theta frequencies, the tonic activity of these neurons, forced by 
optogenetic stimulation should weaken the theta oscillation strength. This explanation is further supported by the results reported by Lu et al. (2020), who increased the power of hippocampal theta oscillations by selectively stimulating the NI neuronal population innervating the MS (and therefore not theta-bursting $\mathrm{NI}$ neurons, as we know from our observations), with light pulses at a frequency and pattern similar to their physiological firing (our study; Lu et al., 2020). The current paper and previous data (Lu et al., 2020; Nunez et al., 2006) indicate that the innervation of the MS by regularly (tonically) firing $\mathrm{NI}$ neurons has a facilitating and/or gating function in the brainstem originating mechanism of theta rhythm induction. In line with these results, Ma et al. (2016), using activation of the $\mathrm{NI}$ via hM3Dq-DREADD, showed that $\mathrm{NI}$ neurons depolarisation - plausibly maintaining their firing pattern - caused an increase in theta power. In the case of the theta-bursting population of neurons, this probably led to the generation of more action potentials in bursts and possible transition of irregularly bursting neurons into the rhythmic theta-bursting firing mode.

Hippocampal theta oscillations result from rhythmic changes in polarisation of the principal cells, induced by the activity of hippocampal interneurons, which in turn are influenced by signals from the medial septum. In our proposed model, two distinct neuronal populations, identified by us in the $\mathrm{NI}$, may be involved in hippocampal theta rhythm generation in different ways: regular, fastfiring theta phase-independent NI neurons innervating the MS may have permissive function, while theta-bursting NI neurons are likely involved in synchronising theta rhythm of other brain structures (Fig. 14). In summary, the results described in this paper shed new light on the interpretation of previous observations and provide the basis for a more precise incorporation of the nucleus incertus in the mechanism of generation and/or modulation of septohippocampal theta oscillations. 
668

669

670

671

672

673

674

675

676

677

678

679

680

681

682

683

684

685

686

687

688

\section{References}

Albert-Gasco H, Ma S, Ros-Bernal F, Sánchez-Pérez AM, Gundlach AL, Olucha-Bordonau FE (2018)

GABAergic Neurons in the Rat Medial Septal Complex Express Relaxin-3 Receptor (RXFP3) mRNA Front Neuroanat. 11:133.

Banerjee A, Shen PJ, Ma S, Bathgate RAD, Gundlach AL (2010) Swim stress excitation of nucleus incertus and rapid induction of relaxin-3 expression via CRF1 activation. Neuropharmacology 58, $145-155$.

Berens P (2009) CircStat: a MATLAB toolbox for circular statistcs. J. Stat. Softw. 31:1-20

Bland BH (1986) The physiology and pharmacology of hippocampal formation theta rhythms. Prog Neurobiol. 26(1):1-54

Bland BH, Konopacki J, Dyck RH (2002) Relationship between membrane potential oscillations and rhythmic discharges in identified hippocampal theta-related cells. J. Neurophysiol., 88:3046-3066.

Borhegyi Z, Varga V, Szilágyi N, Fabo D, Freund TF (2004) Phase segregation of medial septal GABAergic neurons during hippocampal theta activity. J Neurosci. 24(39):8470-9.

Brown RE, McKenna JT (2015) Turning a Negative into a Positive: Ascending GABAergic Control of Cortical Activation and Arousal. Front Neurol. 6:135.

Buzsaki G (1989) Two-stage model of memory trace formation: a role for "noisy" brain states. Neuroscience 31:551-570.

Buzsaki G (2002) Theta oscillations in the hippocampus. Neuron, 33:325-340.

Buzsaki G, Moser El (2013) Memory, navigation and theta rhythm in the hippocampal-entorhinal system. Nat Neurosci. 16(2):130-8 
Cervera-Ferri A, Guerrero-Martínez J, Bataller-Mompeán M, Taberner-Cortes A, Martínez-Ricós J, Ruiz-Torner A, Teruel-Martí V (2011) Theta synchronization between the hippocampus and the nucleus incertus in urethane-anesthetized rats. Exp Brain Res. 211(2):177-92.

Di Prisco GV, Albo Z, Vertes RP, Kocsis B (2002) Discharge properties of neurons of the median raphe nucleus during hippocampal theta rhythm in the rat. Exp. Brain Res. 145(3):383-94.

Goto M, Swanson LW, Canteras NS (2001) Connections of the nucleus incertus. J Comp Neurol. $438: 86-122$.

Haidar M, Guèvremont G, Zhang C, Bathgate RAD, Timofeeva E, Smith CM, Gundlach AL (2017) Relaxin-3 inputs target hippocampal interneurons and deletion of hilar relaxin-3 receptors in "floxedRXFP3" mice impairs spatial memory. Hippocampus. 27(5):529-546.

Haidar M, Tin K, Zhang C, Nategh M, Covita J, Wykes AD, Rogers J, Gundlach AL (2019) Septal GABA and Glutamate Neurons Express RXFP3 mRNA and Depletion of Septal RXFP3 Impaired Spatial Search Strategy and Long-Term Reference Memory in Adult Mice. Front Neuroanat. 13:30.

Hangya B, Borhegyi Z, Szilágyi N, Freund TF, Varga V (2009) GABAergic neurons of the medial septum lead the hippocampal network during theta activity. J Neurosci. 29(25):8094-8102.

Huerta PT, Lisman JE (1993) Heightened synaptic plasticity of hippocampal CA1 neurons during a cholinergically induced rhythmic state. Nature 364:723-725.

Kania A, Szlaga A, Sambak P, Gugula A, Blasiak E, Micioni Di Bonaventura MV, Hossain MA, Cifani C, Hess G, Gundlach AL, Blasiak A (2020) RLN3/RXFP3 Signaling in the PVN Inhibits Magnocellular Neurons via M-like Current Activation and Contributes to Binge Eating Behavior. J Neurosci. 40(28):5362-5375. 
Klapoetke NC, Murata Y, Kim SS, Pulver SR, Birdsey-Benson A, Cho YK, Morimoto TK, Chuong AS, Carpenter EJ, Tian Z, Wang J, Xie Y, Yan Z, Zhang Y, Chow BY, Surek B, Melkonian M, Jayaraman V, Constantine-Paton M, Wong GK, Boyden ES (2014) Independent optical excitation of distinct neural populations. Nat Methods. 11(3):338-46.

Klausberger T, Magill PJ, Márton LF, Roberts JD, Cobden PM, Buzsáki G, Somogyi P (2003) Brain-stateand cell-type-specific firing of hippocampal interneurons in vivo. Nature. 421(6925):844-8.

Kocsis B, Di Prisco GV, Vertes RP (2001) Theta synchronization in the limbic system: the role of Gudden's tegmental nuclei. Eur. J. Neurosci. 13(2):381-388.

Korotkova T, Ponomarenko A, Monaghan CK, Poulter SL, Cacucci F, Wills T, Hasselmo ME, Lever C (2018) Reconciling the different faces of hippocampal theta: The role of theta oscillations in cognitive, emotional and innate behaviors. Neurosci Biobehav Rev. 85:65-80.

Lasztóczi B, Tukker JJ, Somogyi P, Klausberger T (2011) Terminal field and firing selectivity of cholecystokinin-expressing interneurons in the hippocampal CA3 area. J Neurosci. 31(49):18073-93.

Lu L, Ren Y, Yu T, Liu Z, Wang S, Tan L, Zeng J, Feng Q, Lin R, Liu Y, Guo Q, Luo M (2020) Control of locomotor speed, arousal, and hippocampal theta rhythms by the nucleus incertus. Nat Commun. 11(1):262.

Ma S, Allocca G, Ong-Pålsson EK, Singleton CE, Hawkes D, McDougall SJ, Williams SJ, Bathgate RA, Gundlach AL (2017b) Nucleus incertus promotes cortical desynchronization and behavioral arousal. Brain Struct Funct. 222(1):515-537.

Ma S, Blasiak A, Olucha-Bordonau FE, Verberne AJ, Gundlach AL (2013) Heterogeneous responses of nucleus incertus neurons to corticotrophin-releasing factor and coherent activity with hippocampal theta rhythm in the rat. J Physiol. 591:3981-4001. 
Ma S, Hangya B, Leonard CS, Wisden W, Gundlach AL. (2018) Dual-transmitter systems regulating arousal, attention, learning and memory. Neurosci Biobehav Rev. 85:21-33.

Ma S, Olucha-Bordonau FE, Hossain MA, Lin F, Kuei C, Liu C, Wade JD, Sutton SW, Nunez A, Gundlach AL (2009) Modulation of hippocampal theta oscillations and spatial memory by relaxin-3 neurons of the nucleus incertus. Learn Mem. 16:730-742.

Ma S, Smith CM, Blasiak A, Gundlach AL. (2017a) Distribution, physiology and pharmacology of relaxin-3/RXFP3 systems in brain. Br J Pharmacol. 174(10):1034-1048.

Martinez-Bellver S, Cervera-Ferri A, Luque-García A, Martínez-Ricós J, Valverde-Navarro A, Bataller M, Guerrero J, Teruel-Marti V (2017) Causal relationships between neurons of the nucleus incertus and the hippocampal theta activity in the rat. J Physiol. 595(5):1775-1792.

Martinez-Bellver S, Cervera-Ferri A, Martínez-Ricós J, Ruiz-Torner A, Luque-Garcia A, Blasco-Serra A, Guerrero-Martínez J, Bataller-Mompeán M, Teruel-Martí V (2015) Regular theta-firing neurons in the nucleus incertus during sustained hippocampal activation. Eur J Neurosci. 41(8):1049-67.

Nasirova N, Quina LA, Morton G, Walker A, Turner EE (2020) Mapping Cell Types and Efferent Pathways in the Ascending Relaxin-3 System of the Nucleus Incertus. eNeuro. 7(6):ENEURO.027220.2020

Nunez A, Cervera-Ferri A, Olucha-Bordonau F, Ruiz-Torner A, Teruel V (2006) Nucleus incertus contribution to hippocampal theta rhythm generation. Eur J Neurosci. 23(10):2731-8.

Nunez A, Garcia-Austt G, Buno W (1987) Intracellular theta-rhythm generation in identified hippocampal pyramids. Brain Res. 416:289-300.

Oddie SD, Bland BH (1998) Hippocampal formation theta activity and movement selection. Neurosci Biobehav Rev 22:221-231. 
O'Keefe J, Recce ML (1993) Phase relationship between hippocampal place units and the EEG theta rhythm. Hippocampus 3(3):317-30.

Olucha-Bordonau FE, Otero-Garcia M, Sanchez-Perez AM, Nunez A, Ma S, Gundlach AL (2012) Distribution and targets of the relaxin-3 innervation of the septal area in the rat. J Comp Neurol 520:1903-1939.

Olucha-Bordonau FE, Teruel V, Barcia-Gonzalez J, Ruiz-Torner A, Valverde-Navarro AA, MartinezSoriano F (2003) Cytoarchitecture and efferent projections of the nucleus incertus of the rat. J Comp Neurol 464:62-97.

Pachitariu M, Steinmetz N, Kadir S, Carandini M, Harris K (2016) Kilosort: realtime spike-sorting for extracellular electrophysiology with hundreds of channels. bioRxiv. 2016:061481.

Pavlides C, Greenstein YJ, Grudman M, Winson J (1988) Long-term potentiation in the dentate gyrus is induced preferentially on the positive phase of theta-rhythm. Brain Res. 439:383-387.

Paxinos G, Watson C (2007) The rat brain in stereotaxic coordinates, 6th ed. Elsevier, San Diego.

Ryan PJ, Ma S, Olucha-Bordonau FE, Gundlach AL (2011) Nucleus incertus--an emerging modulatory role in arousal, stress and memory. Neurosci Biobehav Rev. 35(6):1326-41.

Strata F (1998) Intrinsic oscillations in CA3 hippocampal pyramids: physiological relevance to theta rhythm generation. Hippocampus, 8:666-679.

Stüttgen M (2020) MLIB - toolbox for analyzing spike data (www.mathworks.com/matlabcentral/fileexchange/37339-mlib-toolbox-for-analyzing-spike-data), MATLAB Central File Exchange. 
774

775

776

777

778

779

780

781

782

Szonyi A, Sos KE, Nyilas R, Schlingloff D, Domonkos A, Takács VT, Pósfai B, Hegedüs P, Priestley JB, Gundlach AL, Gulyás Al, Varga V, Losonczy A, Freund TF, Nyiri G (2019) Brainstem nucleus incertus controls contextual memory formation. Science. 364(6442).

Teruel-Marti V, Cervera-Ferri A, Nunez A, Valverde-Navarro AA, Olucha-Bordonau FE, Ruiz-Torner A (2008) Anatomical evidence for a ponto-septal pathway via the nucleus incertus in the rat. Brain Res 1218: 87-96.

Vertes RP, Hoover WB, Di Prisco GV (2004) Theta rhythm of the hippocampus: subcortical control and functional significance. Behav Cogn Neurosci Rev. 3(3):173-200.

Vertes RP (2005) Hippocampal theta rhythm: a tag for short-term memory. Hippocampus 15:923935.

Watson GS, Williams EJ (1956) On the Construction of Significance Tests on the Circle and the Sphere. Biometrika. 43, 344-352.

Zar JH (1999) Biostatistical Analysis. 4th edition. Prentice Hill.

Zhang F, Wang LP, Boyden ES, Deisseroth K (2006) Channelrhodopsin-2 and optical control of excitable cells. Nat Methods. 3(10):785-92. 
Figures captions

Fig. 1. Histological verification of the positioning of the recording microelectrode array (MEA) in the nucleus incertus ( $\mathrm{NI}$ ) and reconstruction of the location of different types of neurons recorded within the NI. A, left, an example of the Dil (red) filled traces of the four shanks of the MEA (indicated by the white arrows) terminating on the ventral border of the $\mathrm{NI}$ (the brain section is coronal and the MEA penetration was done at a caudal angle of $15^{\circ}$; see Materials and methods section). Right, reconstruction of the location of all recorded NI neurons ( $n=197)$. B, Reconstruction of the location of theta phase-independent neurons (top; $n=117$ ) divided into irregular, slow-firing (middle; $n=71$ ) and regular, fast-firing (bottom; $n=46$ ) neurons. C, location of theta phase-locked (top; $n=80$ ) neurons in the NI divided into bursting (middle; $\mathrm{n}=55$ ) and non-bursting (bottom; $\mathrm{n}=25$ ) neurons. The size of the circles indicates the number of neurons recorded at a given location $(0,0,0,0-1,2,3$, 4 neurons respectively). The dotted line indicates the midline. The location of the $\mathrm{NI}$ neurons is presented on one side, but the recordings were made from both sides of the brain. D, histogram showing the distribution of different types of $\mathrm{NI}$ neurons along anterior-posterior (AP), medial-lateral $(\mathrm{ML})$ and dorsal-ventral (DV) axes (bin $=100 \mu \mathrm{m})$. Scale bars $=200 \mu \mathrm{m}$. Nlc - nucleus incertus pars compacta, NId - nucleus incertus pars dissipata, 4V - 4th ventricle, Cb - cerebellum, DTg - dorsal tegmental nucleus, LC - locus coeruleus, mlf-medial longitudinal fasciculus, RtTg reticulotegmental nucleus of the pons.

Fig. 2. Two types of nucleus incertus neurons that can be distinguished on the basis of their preference to fire action potentials in the hippocampal theta phase-independent or theta phaselocked manner. A, an exemplary $\mathrm{NI}$ neuron that fires action potentials in the theta phaseindependent manner $\left(p_{R}>0.05\right.$ or $\left.p_{R} \leq 0.05 ; r<0.2\right)$. B, an exemplary neuron with strong preference $\left(p_{R}<0.05 ; r \geq 0.2\right)$ to fire at the specific phase of the ongoing hippocampal theta oscillation. Top, scatter plots showing the position of individual action potentials (dots) around two cycles of the 
hippocampal theta oscillation. Middle, circular plots showing the histogram of the angular distribution of action potentials around the cycle of the hippocampal theta oscillation. Red solid lines show direction vectors. The red, dotted line circles indicate the length of the direction vector equal to 0.2 . Bottom, histograms showing the distribution of action potential firing probability around two cycles of the hippocampal theta oscillation. The red, dashed line depicts idealised hippocampal theta oscillation. The trough of the hippocampal theta rhythm was defined as the zero phase (see Materials and methods section). Bins (all histograms) $=10^{\circ}$. In the case of both neurons, analysis of 200s of firing recorded during cortical activation is shown. $r$ - direction vector length; $p_{R}$ - confidence level of Rayleigh's test for uniformity. $\mathbf{C}$ and $\mathbf{D}$, the preference to theta phase of all recorded theta phase-independent $\mathrm{NI}$ neurons and theta phase-locked NI neurons, respectively, during two theta cycles. Top, heat maps of the probability to fire action potentials at a specific hippocampal theta phase for theta phase-independent $(\mathbf{C} ; n=117)$ and theta phase-locked $(\mathbf{D} ; n=80)$ neurons. The probability of action potential generation is colour coded. The neurons are sorted according to the phase of the probability peak. Bottom, the median (blue line) phase preference of the two groups of NI neurons shown above. Light blue area indicates interquartile range; the red, dashed line illustrates idealised hippocampal theta oscillation.

Fig. 3. Electrophysiological types of recorded NI neurons $(n=197)$. Centre, pie chart showing the proportion of the different types in the total $\mathrm{NI}$ neuron population. Left, theta phaseindependent neurons consist of two subgroups: irregular, slow-firing neurons (A) and regular, fastfiring neurons (B). Right, theta phase-locked neurons consist of two subgroups: bursting neurons (C) and non-bursting neurons (D). In each panel, histograms of interspike intervals (left) and autocorrelation function plots (right) during cortical activation are shown for a representative neuron of each type. The theta wave and action potentials generated by an idealised neuron of a given type are schematically presented below the histograms. Bins (all histograms) = $10 \mathrm{~ms}$. 
Fig. 4. The preference of non-bursting and bursting, theta phase-locked NI neurons to fire action potentials at a specific phase of the hippocampal theta. A, circular plot showing the phase preference of individual bursting (blue; $n=55$ ) and non-bursting (green; $n=25$ ) neurons. Solid lines show the mean direction vector of a specific neuronal population (coded by colour). Whiskers represent of the analysed parameters.

circular standard errors of the mean directions. The red, dotted line circle indicates the length of the direction vector equal to 0.2 . Red asterisk denotes a significant phase preference of population of theta bursting $\mathrm{NI}$ neurons, $r$ - direction vector length, $\mathrm{p}_{\mathrm{R}}$-confidence level of Rayleigh's test for uniformity. Heat maps of the probability to fire action potentials at a specific hippocampal theta phase for theta phase-locked bursting (B) and theta phase-locked non-bursting (C) neurons. The neurons are sorted according to the phase of the probability peak. Bottom, the median (blue and green lines) phase preference of the two groups of $\mathrm{NI}$ neurons shown above. Light coloured area indicates interquartile range; the red, dashed line illustrates idealised hippocampal theta oscillation. The trough of the hippocampal theta rhythm was defined as the zero phase.

Fig. 5. The correlation of the parameters describing the strength of the generated bursts (green circles): A, intraburst frequency; $\mathbf{B}$, no. of spikes per burst; $\mathbf{C}$, length of burst, and the intensity of bursting (blue circles): D, bursting rate; $\mathbf{E}$, interburst interval (IBI); F, CV of interburst interval ( $\left.\mathrm{CV}_{\mid \mathrm{BI}}\right)$; G, percent of spikes fired in bursts (\%SFB), with the median firing rate of $\mathrm{NI}$ theta bursting neurons ( $\mathrm{n}$ $=55)$. Asterisks and fitted curves, drawn with a solid line, indicate significant correlation $\left(R^{2} \geq 0.7\right)$, whereas fitted curves, drawn with dotted line, indicate insignificant correlation $\left(R^{2}<0.7\right)$. Individual $R^{2}$ values are given on each plot. Type of fit used is colour coded: linear - violet, exponential - red. Note that in case of insignificant correlations, linear and exponential fits were performed. The blue circles with the whiskers on the side of each graph shows the median, 1st \& 3rd quartiles (Q1 \& Q3) 867 
Fig. 6. Cross-correlation analysis of spontaneous activity, observed during cortical activation, of theta phase-locked NI neurons: A, theta bursting vs. theta bursting (T-bst. vs. T-bst.); B, non-bursting vs. theta bursting (non-bst. vs. T-bst.); C, non-bursting vs. non-bursting (non-bst. vs. non-bst.) and theta phase-independent NI neurons: D, irregular firing vs. irregular firing (irreg. vs. irreg.); E, regular firing vs. irregular firing (reg. vs. irreg.); F, regular firing vs. regular firing (reg. vs. reg.). Top, heatmaps showing cross-correlations of activity of $\mathrm{NI}$ theta bursting (T-bst.) neuron pairs. Cross-correlations are sorted by the time of the peak closest to zero-lag. Cross-correlation with significant peak values (zscore $>2.575$; above $99 \%$ confidence level) are shown first (from top). The bar on the right indicates the proportion of pairs of neurons whose activity was correlated (light red) and uncorrelated (grey). Note, that all theta bursting neuronal pairs were significantly cross-correlated. Middle, times and square roots of z-score values of the cross-correlograms' positive peaks (closest to zero-lag; red dots) and preceding it negative peaks (blue dots). Light grey area indicates range of insignificant z-score values (range \pm 2.575 ; equivalent to $\pm 99 \%$ confidence limits). Bottom, the median (blue line) of all cross-correlograms shown above. Light blue area indicates interquartile range.

Fig. 7. Two types of nucleus incertus neurons that can be distinguished on the basis of their preference to fire action potentials in the hippocampal delta wave-independent or delta wave-locked manner. A, an exemplary $\mathrm{NI}$ neuron that fires action potentials in the delta wave-independent manner (peak z-score of delta wave zero-phase triggered PSTH < 1.96). B, an exemplary neuron with strong preference to fire in a hippocampal delta wave-wave locked manner (peak z-score of delta wave zero-phase triggered PSTH peak $\geq 1.96)$. Top, scatter plots showing the position of individual action potentials (dots) around delta wave negative peak. Bottom, histograms showing the distribution of action potential firing around delta wave negative peak. The red, dashed line depicts idealised hippocampal delta wave. Light grey area indicates range of insignificant z-score values (range \pm 1.96 ; equivalent to $\pm 95 \%$ confidence limits). Bins $=10 \mathrm{~ms}$. C and $\mathbf{D}$, The relation between firing of all recorded delta wave-independent (C) and delta wave-locked (D) NI neurons and negative 
peak of hippocampal CA1 delta waves. Top, heat maps showing z-score values of delta wave zerophase triggered PSTH (10ms bins) for delta wave-independent (C; $n=144)$ and delta wave-locked (D; $n=53)$ neurons. The $z$-score values are colour coded. The neurons are sorted according to the time of the z-value peak. Bottom, the median (blue line) delta wave zero-phase triggered PSTH of the two groups of NI neurons shown above. Light blue area indicates interquartile range; the red, dashed line illustrates idealised hippocampal delta wave.

Fig. 8. Electrophysiological types of recorded NI neurons $(n=197)$. Centre, pie chart showing the proportion of the different types in the total NI neuron population. Left, delta wave-independent neurons consist of two subgroups: irregular, slow-firing neurons and regular, fast-firing neurons. Right, delta wave-locked neurons consist of two subgroups: non-bursting neurons and bursting neurons. In each panel, histograms of interspike intervals (left) and autocorrelation function plots (right) during hippocampal SWA are shown for a representative neuron of each type. Fragment of hippocampal LFP is shown at the bottom of each panel. Vertical, lines indicate action potentials generated by simultaneously recorded different types of NI neurons. Bins (all histograms) = $10 \mathrm{~ms}$.

Fig. 9. Cross-correlation analysis of spontaneous activity, observed during cortical SWA, of delta wave-locked NI neurons: A, delta bursting vs. delta bursting (D-bst. vs. D-bst.); B, non-bursting vs. delta bursting (non-bst. vs. D-bst.); C, non-bursting vs. non-bursting (non-bst. vs. non-bst.) and delta wave-independent NI neurons: D, irregular firing vs. irregular firing (irreg. vs. irreg.); E, regular firing vs. irregular firing (reg. vs. irreg.); F, regular firing vs. regular firing (reg. vs. reg.). Top, heatmaps showing cross-correlations of activity of $\mathrm{NI}$ theta bursting (T-bst.) neuron pairs. Cross-correlations are sorted by the time of the peak closest to zero-lag. Cross-correlation with significant peak values (zscore $>2.575$; above $99 \%$ confidence level) are shown first (from top). The bar on the right indicates the proportion of pairs of neurons whose activity was correlated (light red) and uncorrelated (grey). Note, that all theta bursting neuronal pairs were significantly cross-correlated. Middle, times and 
square roots of z-score values of the cross-correlograms' positive peaks (closest to zero-lag; red dots) and preceding it negative peaks (blue dots). Light grey area indicates range of insignificant z-score values (range \pm 2.575 ; equivalent to $\pm 99 \%$ confidence limits). Bottom, the median (blue line) of all cross-correlograms shown above. Light blue area indicates interquartile range.

Fig. 10. Responses of different types of $\mathrm{NI}$ neurons to the optogenetic stimulation of the MS during hippocampal theta ans SWA. A, Schemes illustrating the injection of viral vector into the MS and the experimental procedure conducted 3 weeks after the injection. B, Histological verification of the positioning of the optical fibre above the medial septum and the recording microelectrode array (MEA) in the nucleus incertus. Top, an example of the Dil (red) filled trace of the optical fibre terminating above the MS transfected with the viral vector (green). aca - anterior commissure, anterior part, cc - corpus callosum, Ctx - cerebral cortex, CPu - caudate putamen, LV - lateral ventricle, MS - medial septal nucleus. Bottom, an example of the Dil (red) filled traces of the four shanks of the MEA. Light grey spots represent electrodes positioned within the NI, whereas dark grey spots are localised outside the nucleus incertus. NIc - nucleus incertus pars compacta, NId - nucleus incertus pars dissipata, 4V - 4th ventricle, $\mathrm{Cb}$ - cerebellum, DTg - dorsal tegmental nucleus, mlf medial longitudinal fasciculus. C and $\mathbf{D}$, heatmaps showing the responses of different types of $\mathrm{NI}$ neurons to MS stimulation during cortical activation and SWA, respectively. The values of PSTHS (10ms bins; normalized to baseline) are colour coded. Vertical dotted lines indicate MS stimulation onset and offset (laser light: $473 \mathrm{~nm},<10 \mathrm{~mW} ; 50 \mathrm{~ms}$ ). Light blue, bright red, and gray bars to the right of the heatmaps indicate the proportion of inhibited, excited and non-responding neurons, respectively. $\mathbf{E}$ and $\mathbf{F}$, response of selected populations of $\mathrm{NI}$ neurons to $\mathrm{MS}$ stimulation during cortical activation and SWA, respectively. Responses of theta-locked, theta-independent, theta bursting, delta-wave-locked and delta wave-independent populations of NI neurons are shown. Blue line - median PSTH value; light blue area indicates interquartile range; cyan bar indicates the stimulation time. G, Pie chart showing the proportions of response types of NI neurons whose 
responses to MS stimulation were observed in both cortical states (blue: inhibition, red: excitation, gray: no response).

Fig. 11. A, scatter plots (top) and histograms (bottom) showing the exemplary response of thetabursting $\mathrm{NI}$ neuron to an optogenetic stimulation at three different theta frequencies $(4,8$ and 12 $\mathrm{Hz}$ ). Note that in all cases the neuron reacted with inhibition followed by a rebound excitation. B, responses of the same $\mathrm{NI}$ theta-bursting neuron to high-frequency stimulation of the MS $(20 \mathrm{~Hz})$. Representative fragments of the raw signal and separated spikes (red) of the recorded NI neuron. C, histograms of interspike intervals (top) and autocorrelation function plots (bottom) of exemplary theta-bursting NI neuron (shown on A and B) before (left), during (middle; marked with light blue colour) and after (right) the high frequency, optogenetic stimulation of the MS. Cyan coloured bars indicate the laser light $(473 \mathrm{~nm} ;<10 \mathrm{~mW})$ pulses. Bins (all histograms) $=10 \mathrm{~ms}$.

Fig. 12. Ex vivo responses of the NI neurons to optogenetic stimulation of the medial septum (MS) originating axonal endings. A, schematic representation of the experimental design showing viral vectors injection procedure and subsequent MS axonal endings optogenetic stimulation and wholecell voltage clamp recording of the NI neurons. B, Reconstruction of the location of biocytin filled NI neurons in which iPSCs (blue circles), ePSCs (red circle) or no change (grey circles) in response to optogenetic stimulation of the MS originating axonal endings were recorded. C, Average $( \pm$ SD) iPSC (20 stimulations) recorded from the $\mathrm{NI}$ neuron following optogenetic stimulation of MS axonal endings at command potential of $-50 \mathrm{mV}$ and its disappearance at $-90 \mathrm{mV}$. D, Average ( \pm SD) iPSC (20 stimulations) recorded from the $\mathrm{NI}$ neuron following optogenetic stimulation of MS axonal endings under control conditions (SACSF), its complete disappearance in the presence of $G_{A B A}$ receptor antagonist - gabazine $(5 \mu \mathrm{M})$ and partial return after drug washout. E, Distribution of iPSCs' 
amplitudes in the control conditions (SACSF), during gabazine application and washout $(n=7)$. Cyan coloured bars indicate the laser light pulses (465 nm; 5 ms, 10 s interval; <10 mW).

Fig. 13. Identification of nucleus incertus neurons innervating the medial septum. A, schemes illustrating the injection of retrograde viral vector into the MS and the experimental procedure conducted 3 weeks after the injection. B, fragments of raw signal showing responses to different optogenetic stimulation protocols: single pulse lasting $50 \mathrm{~ms}$ (top) and short, $5 \mathrm{~ms}$ pulses at increasing frequencies $(10,30$ and $50 \mathrm{~Hz})$. C, overlaid averaged spike waveforms from the baseline (black) and from the periods of light stimulation (cyan). D, histogram of interspike intervals (left) and autocorrelation function plot (right) of exemplary theta phase-independent, regular, fast firing $\mathrm{NI}$ neuron that was optogenetically identified as MS projecting (shown on B and C). Bins = 10ms. Cyan coloured bars indicate the laser light pulses (473 nm; $<10 \mathrm{~mW})$.

Fig. 14. The postulated nucleus incertus contribution to hippocampal theta rhythm generation. A, a simplified diagram showing the nuclei of the septohippocampal system, the nucleus incertus and their interconnections. The abbreviations inside the ovals indicate the cell type: ACh - cholinergic, GABA - GABAergic, Glu - glutamatergic within medial septum/vertical limb of the diagonal band nucleus (MS/DBv), PC - pyramidal cell, BASK - basket cell, OLM - oriens-lacunosum-moleculare cell within the hippocampus and Fast Reg - regular, fast-firing theta phase-independent, Slow Irreg irregular, slow-firing theta phase-independent, BST - bursting, theta phase-locked, non-BST - nonbursting, theta phase-locked within the nucleus incertus. The arrows endings indicate excitatory and the perpendicular endings indicate inhibitory connections. B, Idealised hippocampal theta oscillation observed in the stratum lacunosum moleculare of the hippocampal CA1 region and the schematic temporal distribution of firing of theta-bursting neurons in: medial septum (blue and green bars), nucleus incertus (purple bars), hippocampal OLM cells (orange bars) and hippocampal basket cell (cyan bars). 
Tab. 1. Firing of different types of NI neurons during state of cortical activation.

\begin{tabular}{|c|c|c|c|c|c|c|c|c|}
\hline & & & $\begin{array}{c}\text { median FR } \\
{[\mathrm{Hz}]}\end{array}$ & $\begin{array}{c}1^{\text {st }} ; 3 \text { rd quartile } \\
{[\mathrm{Hz}]}\end{array}$ & $\begin{array}{c}\text { Mann-Whitney } \\
\text { test }\end{array}$ & CV & $\begin{array}{l}1^{\text {st }} ; 3 \text { rd } \\
\text { quartile }\end{array}$ & $\begin{array}{c}\text { Mann-Whitney } \\
\text { test }\end{array}$ \\
\hline \multirow{4}{*}{ 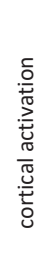 } & $\frac{\circ}{\frac{0}{0}}$ & $\begin{array}{c}\text { irregular, } \\
\text { slow-firing }(n=71)\end{array}$ & 7.7 & $5.2 ; 17.6$ & \multirow{2}{*}{$\begin{array}{c}\mathrm{p}<0.0001 * \\
\mathrm{U}=543.0\end{array}$} & 0.68 & $0.51 ; 0.9$ & \multirow{2}{*}{$\begin{array}{c}p<0.0001^{*} \\
U=0.0\end{array}$} \\
\hline & 홍 & $\begin{array}{c}\text { regular, } \\
\text { fast-firing }(n=46)\end{array}$ & 26.5 & $16.0 ; 37.4$ & & 0.19 & $0.17 ; 0.21$ & \\
\hline & \multirow{2}{*}{ 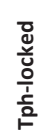 } & $\begin{array}{l}\text { bursting } \\
(n=55)\end{array}$ & 42.6 & $27.5 ; 59.7$ & \multirow{2}{*}{$\begin{array}{c}\mathrm{p}<0.0001^{*} \\
U=39.0\end{array}$} & 1.1 & $0.9 ; 1.34$ & \multirow{2}{*}{$\begin{array}{c}\mathrm{p}<0.0001^{*} \\
U=319.5\end{array}$} \\
\hline & & $\begin{array}{c}\text { non-bursting } \\
(n=25)\end{array}$ & 5.5 & $4.5 ; 11.6$ & & 0.8 & $0.7 ; 1.0$ & \\
\hline
\end{tabular}

Tab. 2. Firing of different types of NI neurons during state of cortical SWA.

\begin{tabular}{|c|c|c|c|c|c|c|c|c|}
\hline & & & $\begin{array}{c}\text { median FR } \\
{[\mathrm{Hz}]}\end{array}$ & $\begin{array}{c}1^{\text {st }} ; 3 \text { rd quartile } \\
{[\mathrm{Hz}]}\end{array}$ & $\begin{array}{c}\text { Mann-Whitney } \\
\text { test }\end{array}$ & CV & $\begin{array}{l}1^{\text {st }} ; 3 \text { rd } \\
\text { quartile }\end{array}$ & $\begin{array}{c}\text { Mann-Whitney } \\
\text { test }\end{array}$ \\
\hline \multirow{4}{*}{ 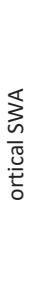 } & $\frac{\dot{0}}{\frac{0}{0}}$ & $\begin{array}{c}\text { irregular, } \\
\text { slow-firing }(n=118)\end{array}$ & 6.5 & $3.0 ; 12.6$ & \multirow{2}{*}{$\begin{array}{c}\mathrm{p}<0.0001^{*} \\
\mathrm{U}=317.0\end{array}$} & 1.0 & $0.84 ; 1.4$ & \multirow{2}{*}{$\begin{array}{c}\mathrm{p}<0.0001^{*} \\
\mathrm{U}=0.0\end{array}$} \\
\hline & ڤั่ & $\begin{array}{c}\text { regular, } \\
\text { fast-firing }(n=26)\end{array}$ & 29.4 & $24.1 ; 35.2$ & & 0.17 & $0.15 ; 0.19$ & \\
\hline & $\begin{array}{l}\text { ரृ } \\
\text { 흔 }\end{array}$ & $\begin{array}{l}\text { bursting } \\
(n=20)\end{array}$ & 20.8 & $17.4 ; 33.1$ & \multirow{2}{*}{$\begin{array}{c}p<0.0001 * \\
U=106.0\end{array}$} & 1.1 & $1.0 ; 1.2$ & \multirow{2}{*}{$\begin{array}{l}\mathrm{p}=0.012 \\
\mathrm{U}=194.0\end{array}$} \\
\hline & à & $\begin{array}{c}\text { non-bursting } \\
(n=33)\end{array}$ & 9.0 & $3.8 ; 15.5$ & & 0.9 & $0.76 ; 1.1$ & \\
\hline
\end{tabular}

Tab. 3. Parameters of NI neurons' responses to the medial septum optogenetic stimulation.

\begin{tabular}{|c|c|c|c|c|c|c|c|}
\hline & & & $\begin{array}{l}\text { response } \\
\text { type }\end{array}$ & $\begin{array}{l}\text { fraction } \\
\text { (count) }\end{array}$ & $\begin{array}{c}\text { FR change } \\
{[\%]}\end{array}$ & $\begin{array}{l}\text { latency to max } \\
\text { [ms] }\end{array}$ & $\begin{array}{l}\text { duration } \\
\text { [ms] }\end{array}$ \\
\hline \multirow{12}{*}{ 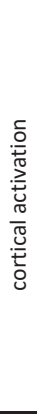 } & \multirow{6}{*}{ 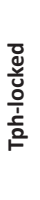 } & \multirow{3}{*}{$\begin{array}{l}\text { bursting } \\
(n=7)\end{array}$} & inhib & $86 \%(6)$ & $-45(-53 ;-41)$ & $42.5(35 ; 85.3)$ & $120(107 ; 151.5)$ \\
\hline & & & excit & $0 \%$ & - & - & - \\
\hline & & & no resp. & $14 \%(1)$ & - & - & - \\
\hline & & \multirow{3}{*}{$\begin{array}{l}\text { non-bursting } \\
\qquad(n=5)\end{array}$} & inhib & $40 \%(2)$ & -65 & 57 & 142.5 \\
\hline & & & excit & $40 \%(2)$ & 76 & 46 & 104.5 \\
\hline & & & no resp. & $20 \%(1)$ & - & - & - \\
\hline & \multirow{6}{*}{ 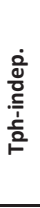 } & \multirow{3}{*}{$\begin{array}{c}\text { irregular, } \\
(n=16)\end{array}$} & inhib & $31 \%(5)$ & $-58(-71 ;-25)$ & $68(34.5 ; 91)$ & $131(87.5 ; 188)$ \\
\hline & & & excit & $25 \%(4)$ & $63(43 ; 99)$ & $51.5(47.8 ; 102.5)$ & 154 (99.8; 273.5) \\
\hline & & & no resp. & $44 \%(7)$ & - & - & - \\
\hline & & \multirow{3}{*}{$\begin{array}{r}\text { regular, } \\
(n=16)\end{array}$} & inhib & $25 \%(4)$ & $-12(-32 ;-5)$ & $38.5(25.3 ; 51.8)$ & $126(106.8 ; 139.3)$ \\
\hline & & & excit & $19 \%(3)$ & $25(11 ; 33)$ & $29(23 ; 46)$ & $118(103 ; 123)$ \\
\hline & & & no resp. & $56 \%(9)$ & - & - & - \\
\hline \multirow{12}{*}{ 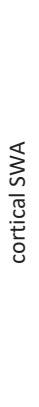 } & \multirow{6}{*}{ 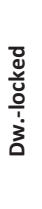 } & \multirow{3}{*}{$\begin{array}{l}\text { bursting } \\
(n=4)\end{array}$} & inhib & $75 \%(3)$ & $-74(-74 ;-54)$ & $92(48 ; 109)$ & $132(110 ; 140)$ \\
\hline & & & excit & $25 \%(1)$ & 74 & 63 & 128 \\
\hline & & & no resp. & $0 \%$ & - & - & - \\
\hline & & \multirow{3}{*}{$\begin{array}{l}\text { non-bursting } \\
\quad(n=4)\end{array}$} & inhib & $0 \%$ & - & - & - \\
\hline & & & excit & $50 \%(2)$ & 77 & 40.5 & 173 \\
\hline & & & no resp. & $50 \%(2)$ & - & - & - \\
\hline & \multirow{6}{*}{ 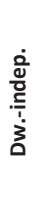 } & \multirow{3}{*}{$\begin{array}{l}\text { irregular, } \\
(n=20)\end{array}$} & inhib & $25 \%(5)$ & $-63(-75 ;-33)$ & $46(38 ; 56)$ & $108(105.5 ; 125.5)$ \\
\hline & & & excit & $15 \%(3)$ & $104(102 ; 106)$ & $54(36 ; 123)$ & $121(103 ; 294)$ \\
\hline & & & no resp. & $60 \%(12)$ & - & - & - \\
\hline & & \multirow{3}{*}{$\begin{array}{l}\text { regular, } \\
(n=9)\end{array}$} & inhib & $44 \%(4)$ & $-17(-20 ;-7)$ & $48(30.8 ; 63)$ & $128(79.3 ; 162.5)$ \\
\hline & & & excit & $11 \%(1)$ & 14 & 30 & 122 \\
\hline & & & no resp. & $45 \%(4)$ & - & - & - \\
\hline
\end{tabular}



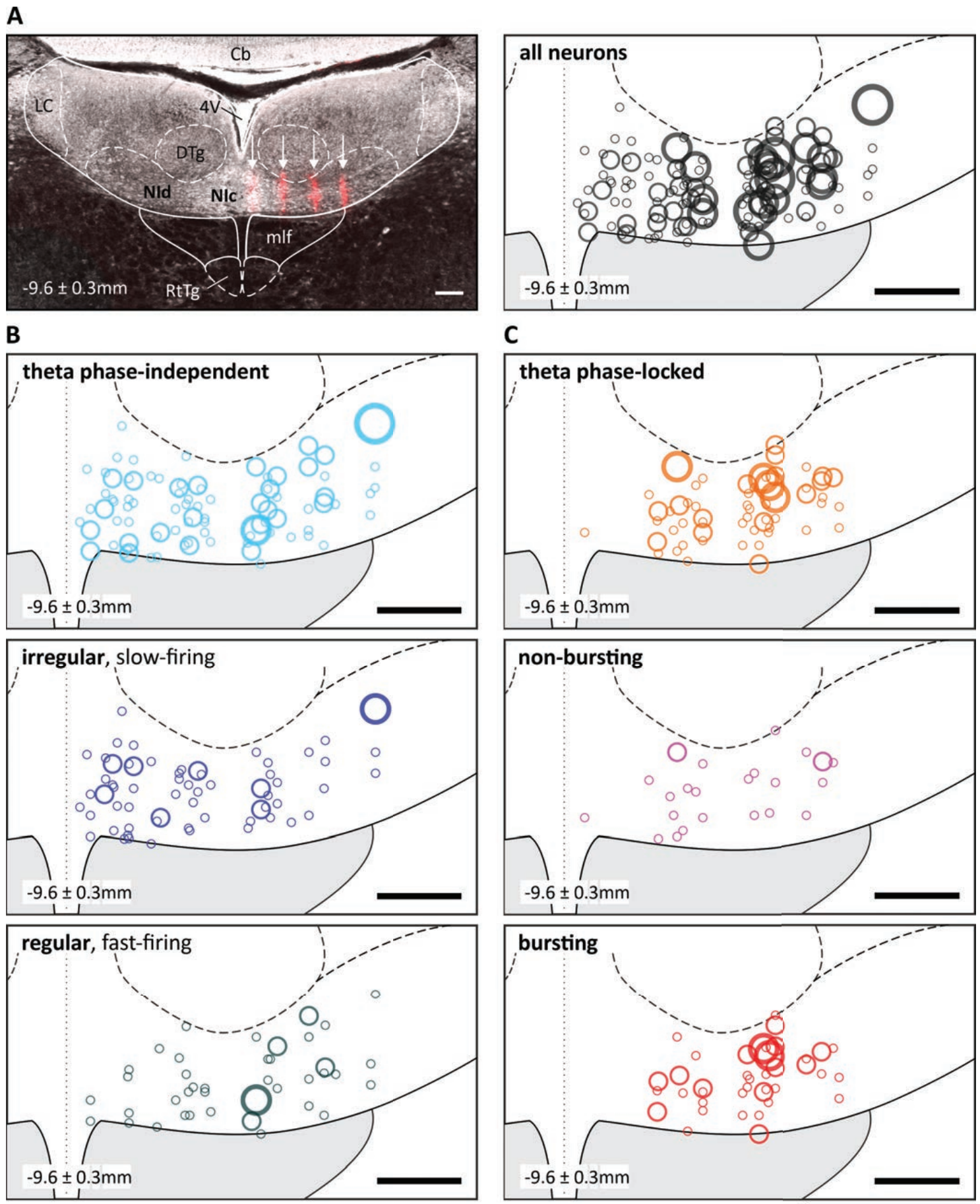

D $\square$ irregular, slow-firing $\square$ regular, fast-firing $\square$ non-bursting $\square$ bursting
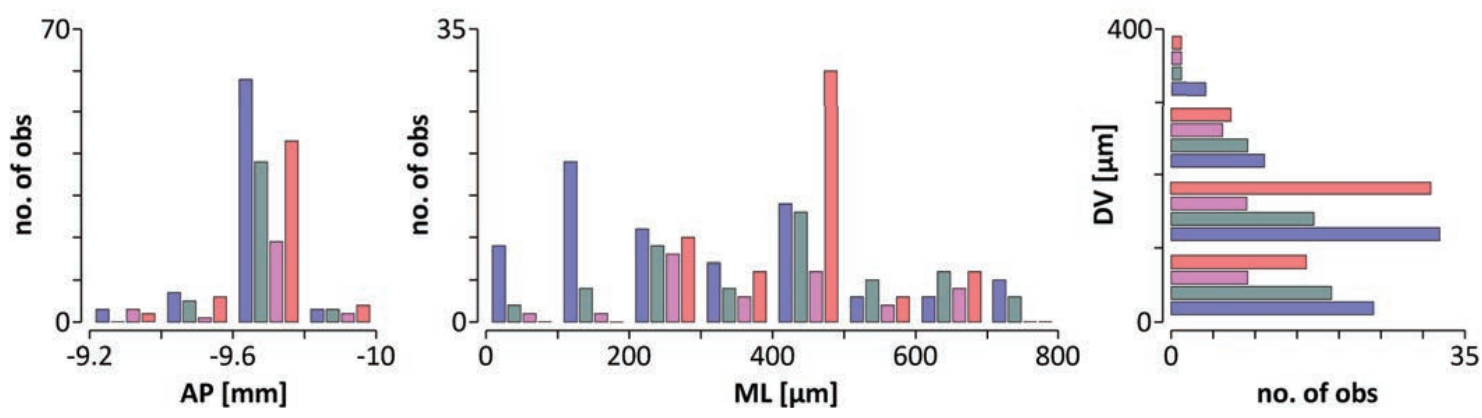


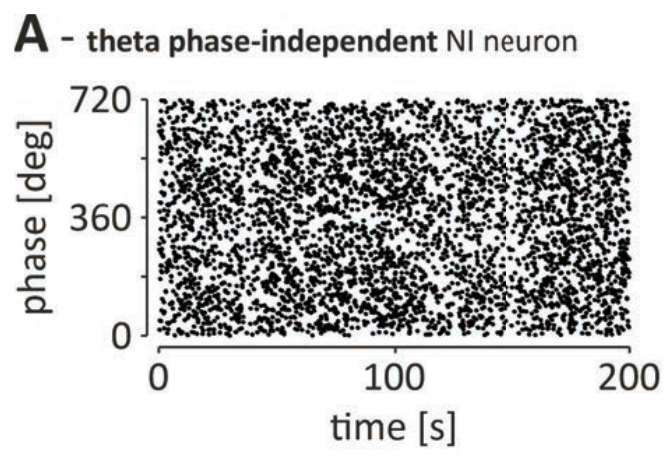

B - theta phase-locked NI neuron
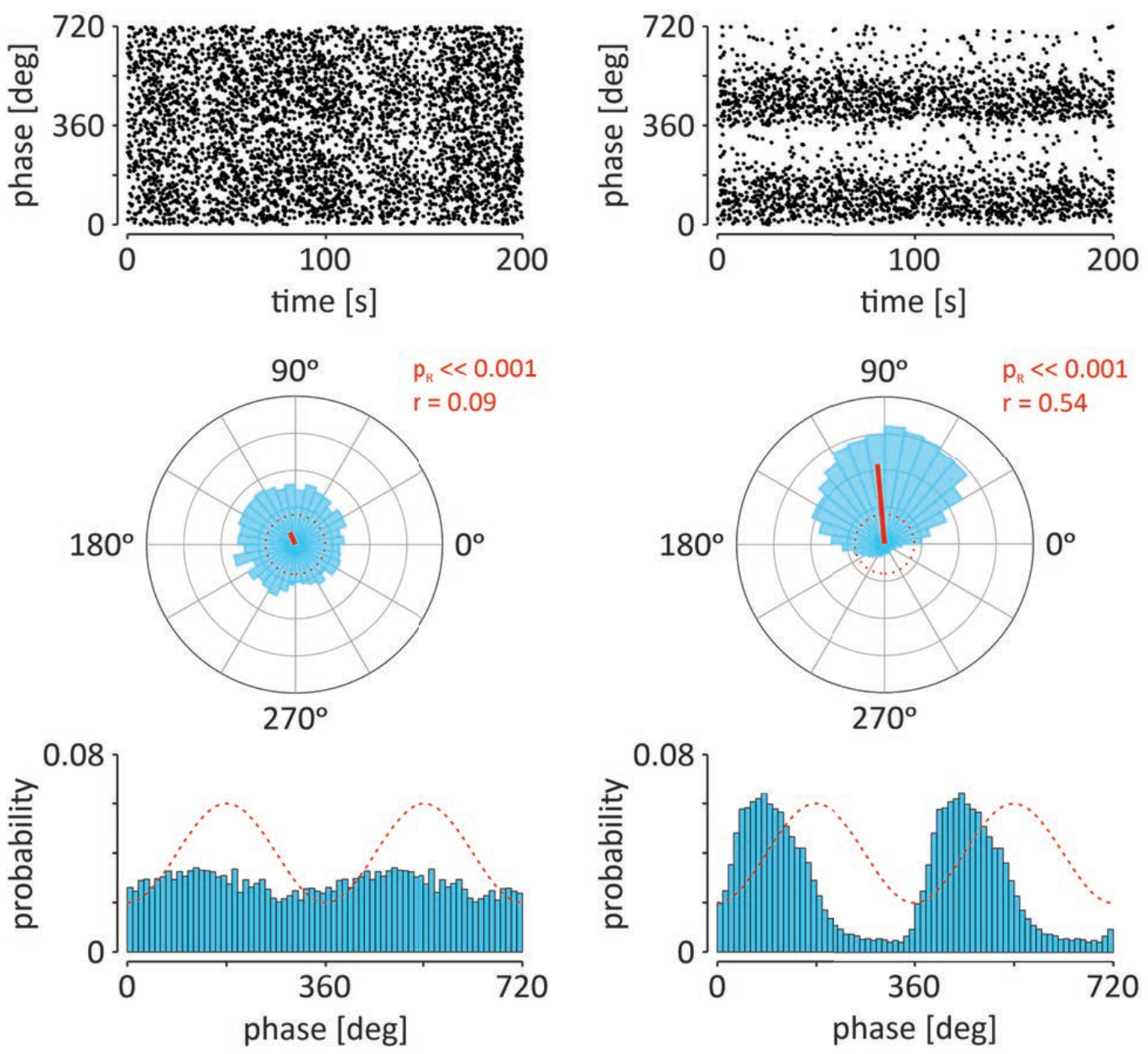

C - theta phase-independent $\mathrm{NI}$ neurons

D - theta phase-locked NI neurons
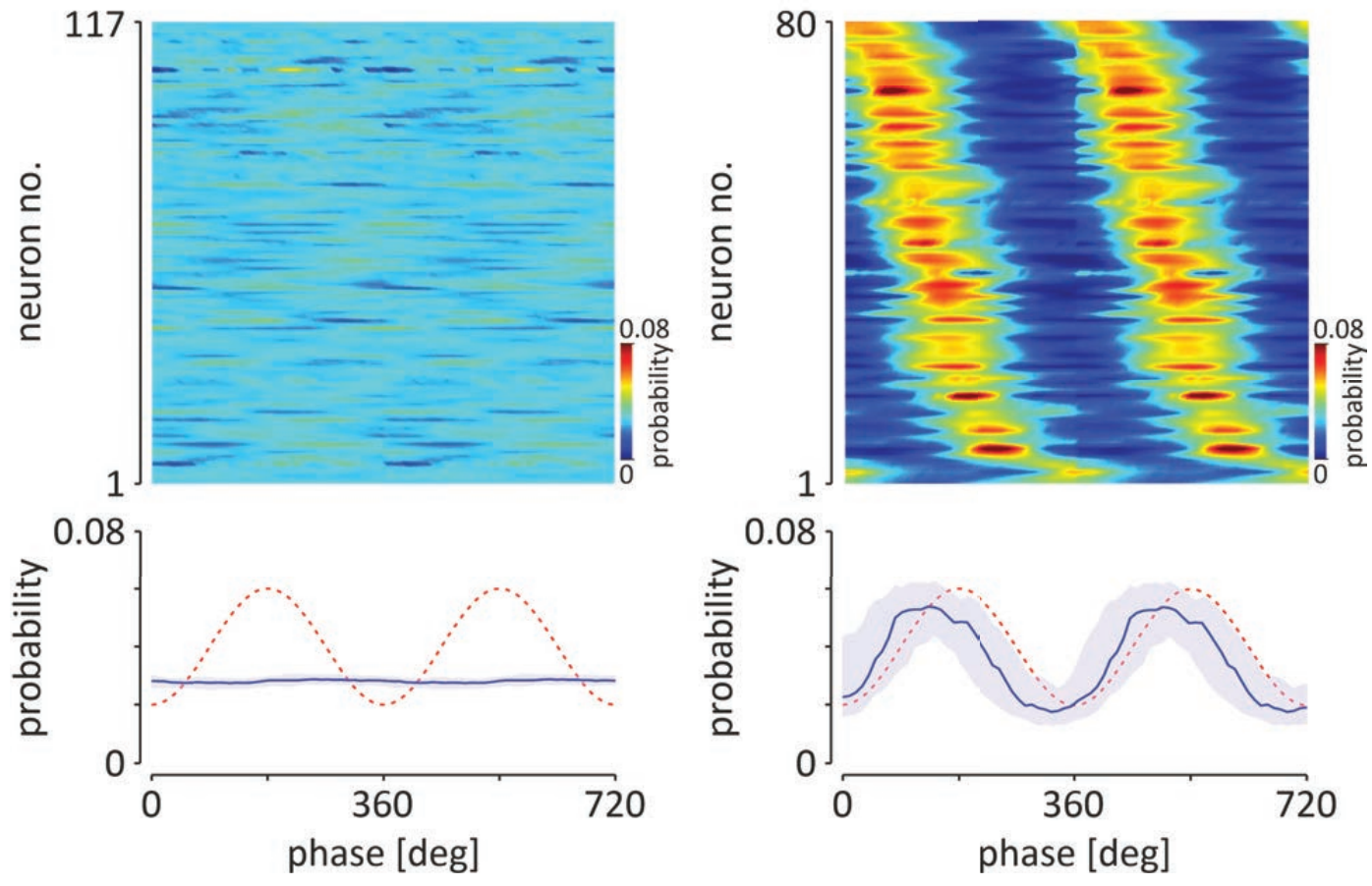
theta phase-independent NI neurons: 59\% ( $n=117)$

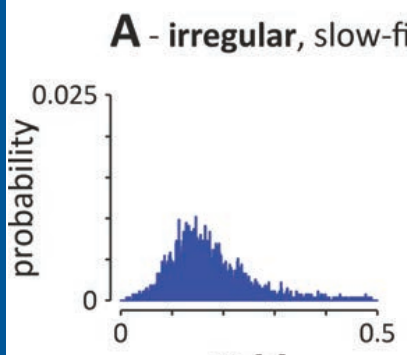

ISI [s]

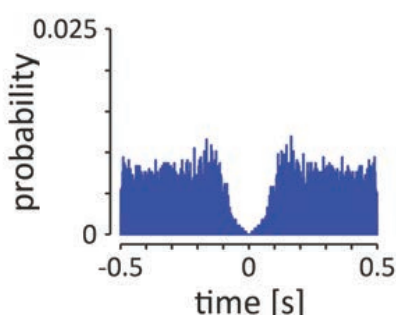

time [s] theta phase-locked NI neurons: $41 \%(n=80)$

\section{C - bursting}
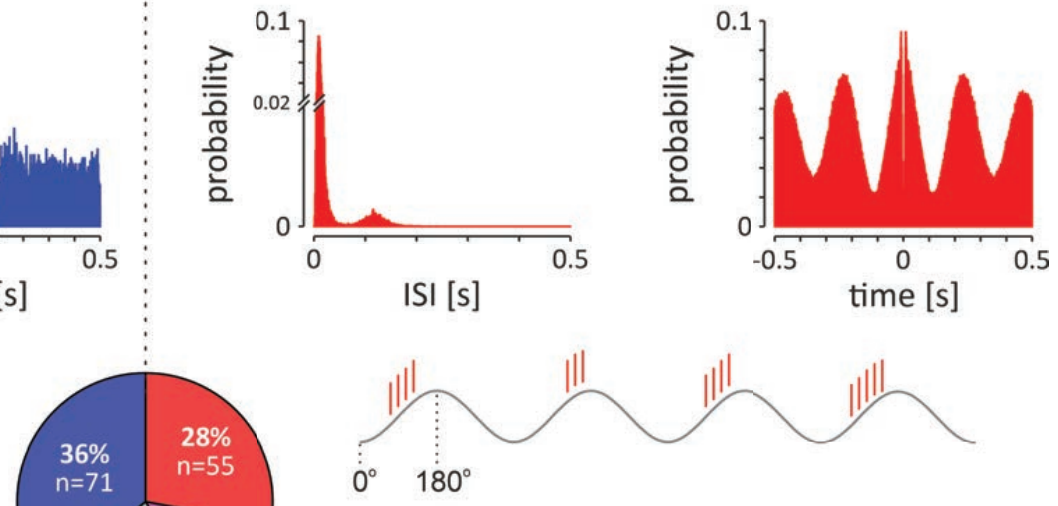

$13 \%$

D - non-bursting

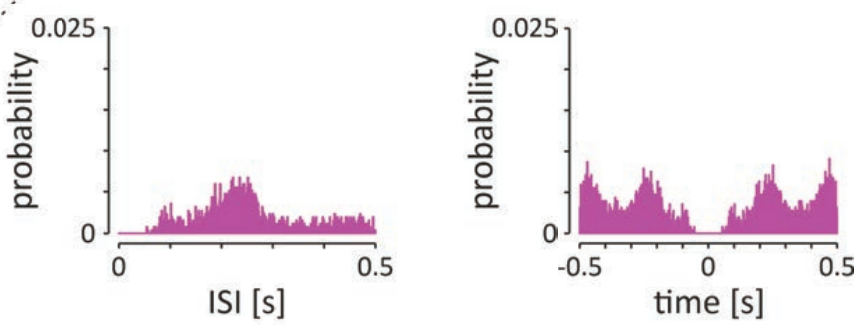

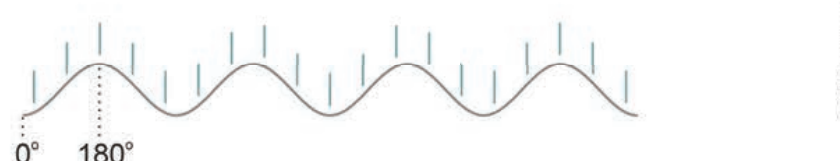

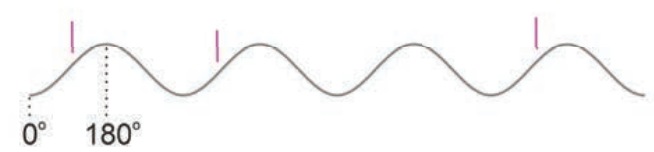


A

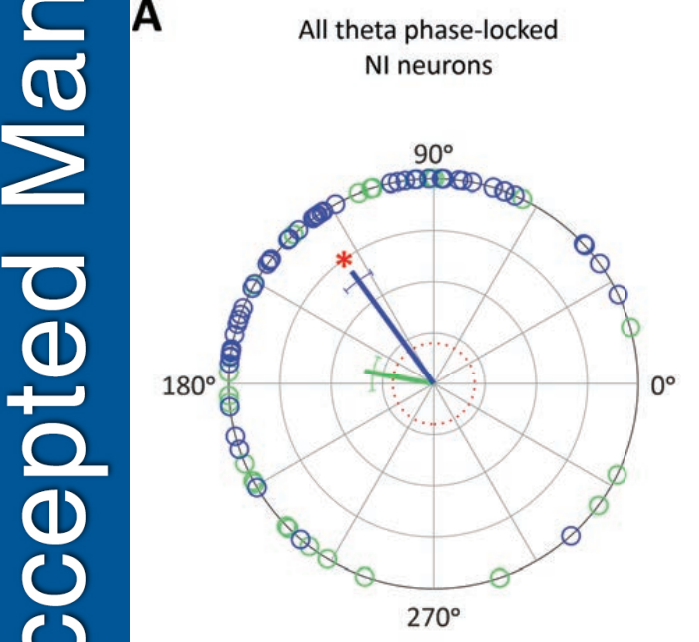

( $<$

O bursting $(n=55)$

$r=0.70, p_{R} \ll<0.001$

non-bursting ( $n=25$ ) $r=0.34, p_{n}=0.051$

B
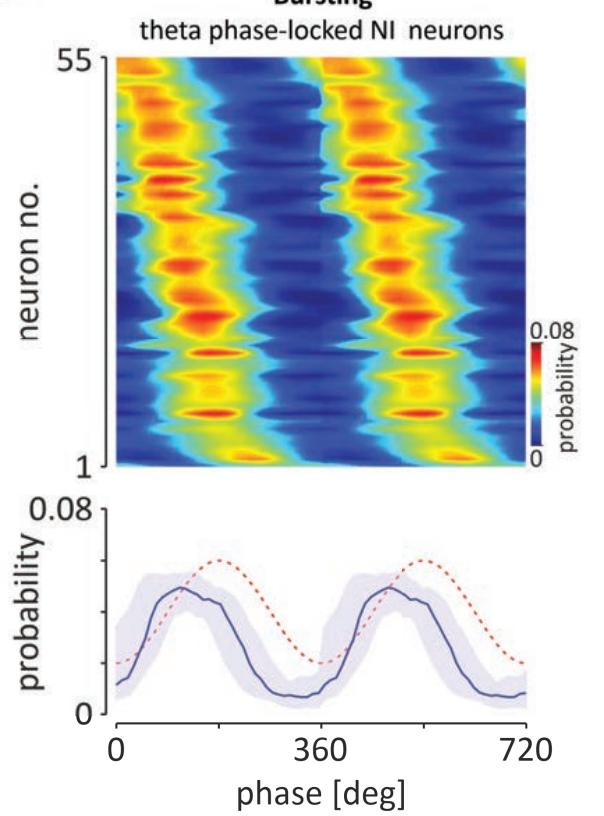

C

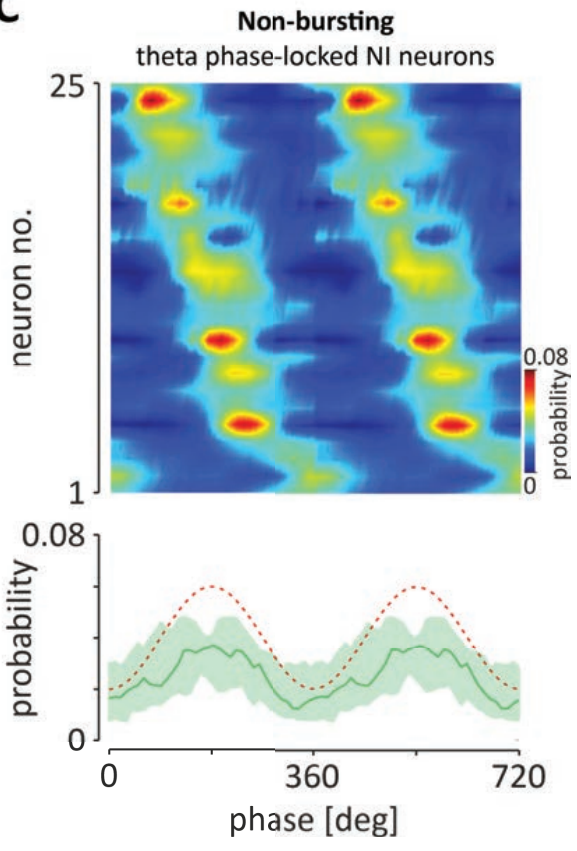




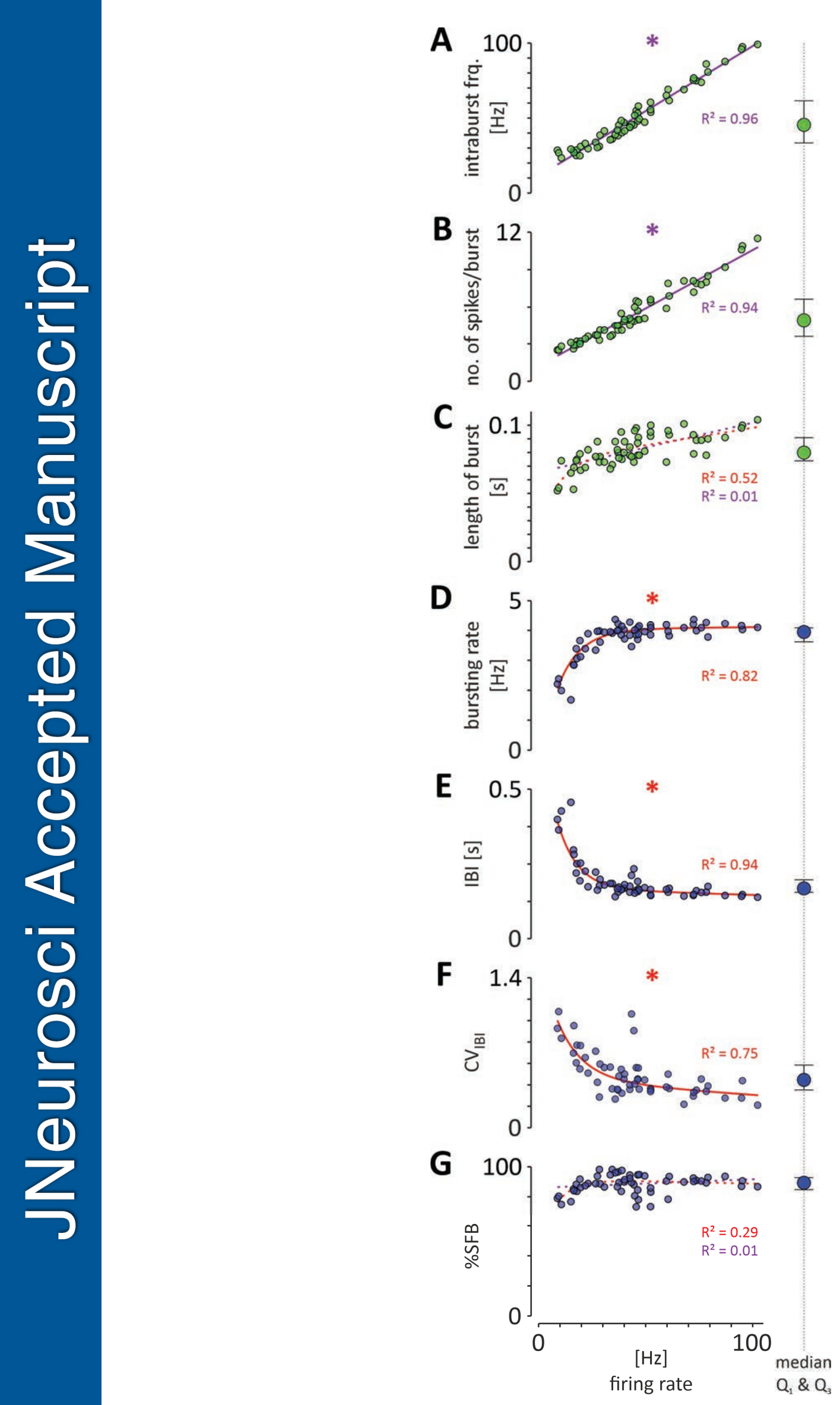


Theta phase-locked NI neurons

A T-bst. vs. T-bst.
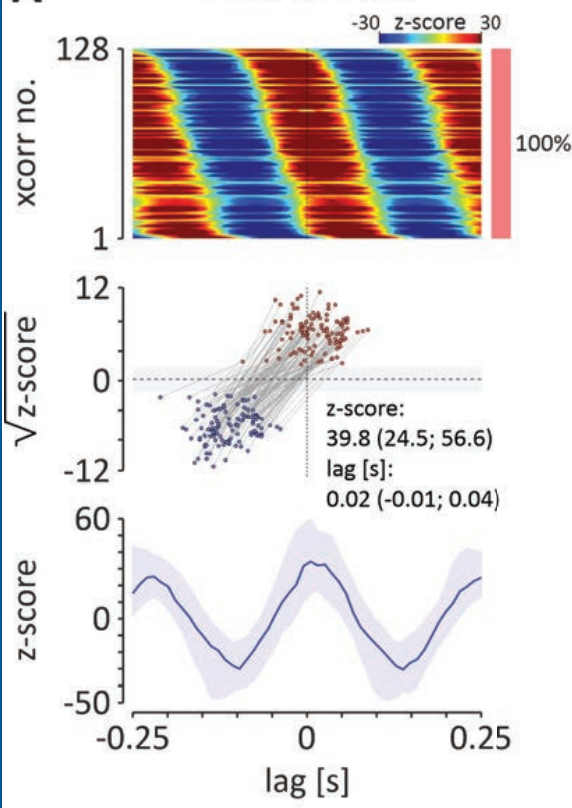

B
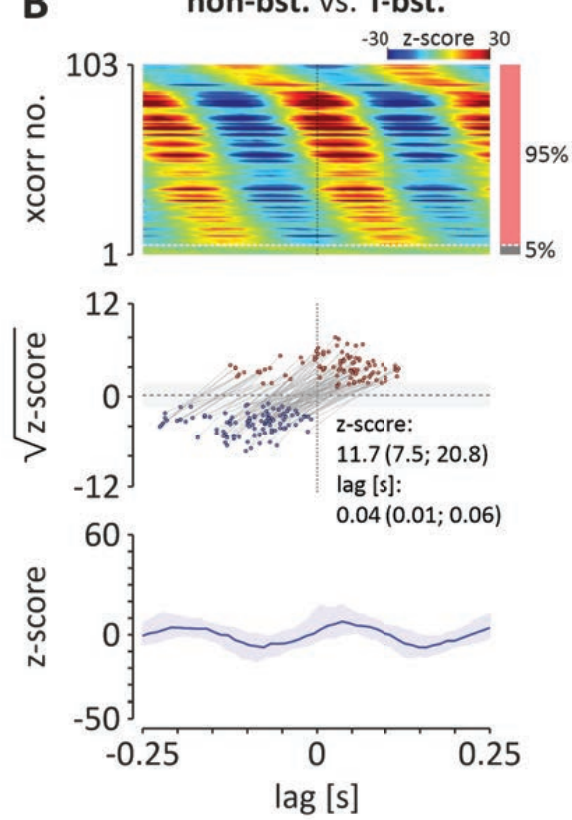

C non-bst. vs. non-bst.
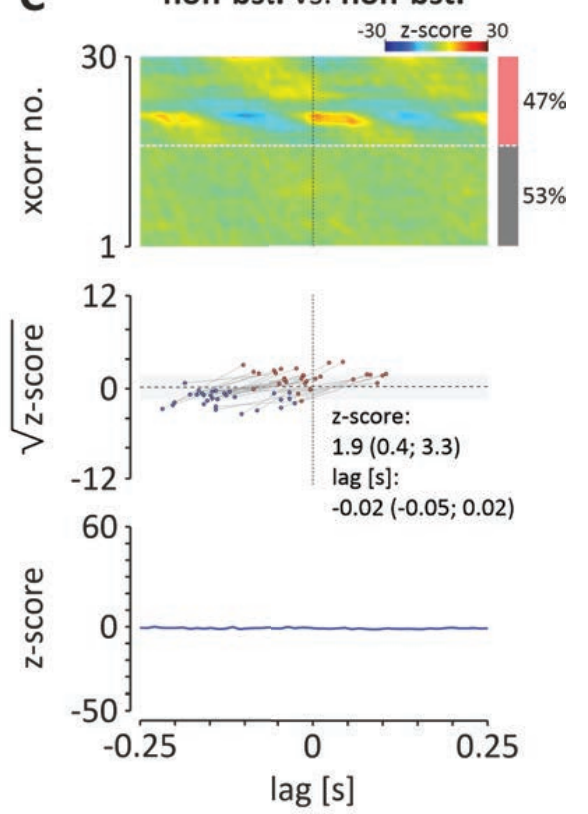

Theta phase-independent NI neurons

D irreg. vs. irreg. E
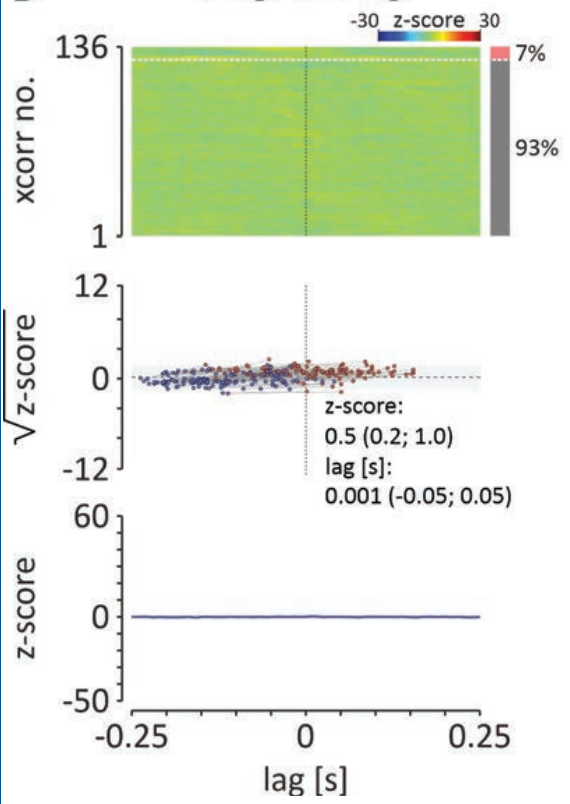

E reg. vs. irreg.
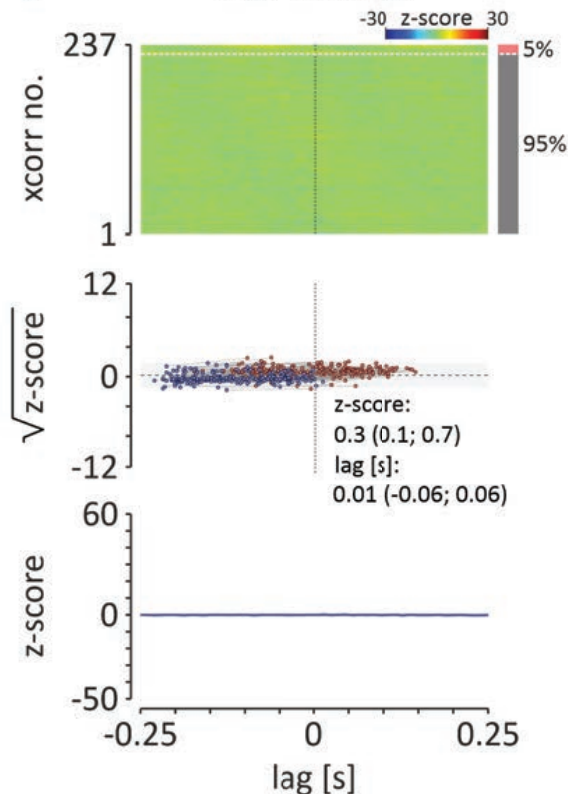

F reg. vs. reg.
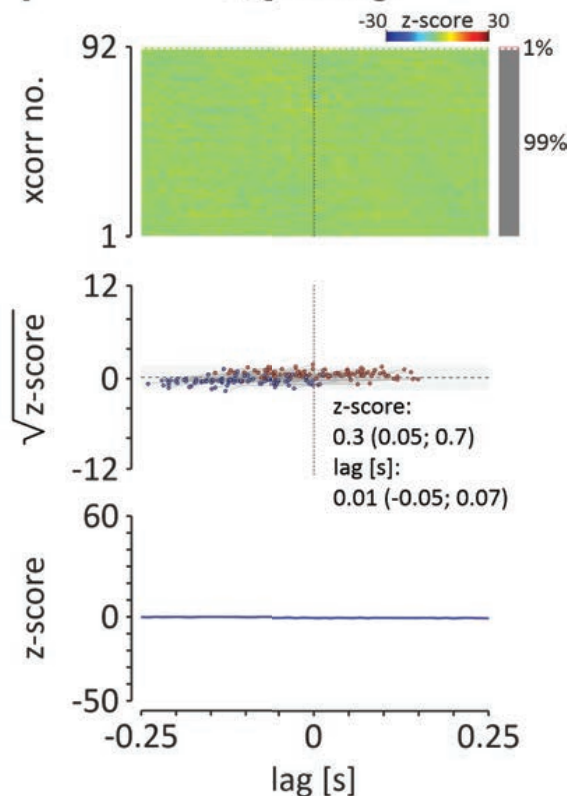
A - delta wave-independent $\mathrm{NI}$ neuron
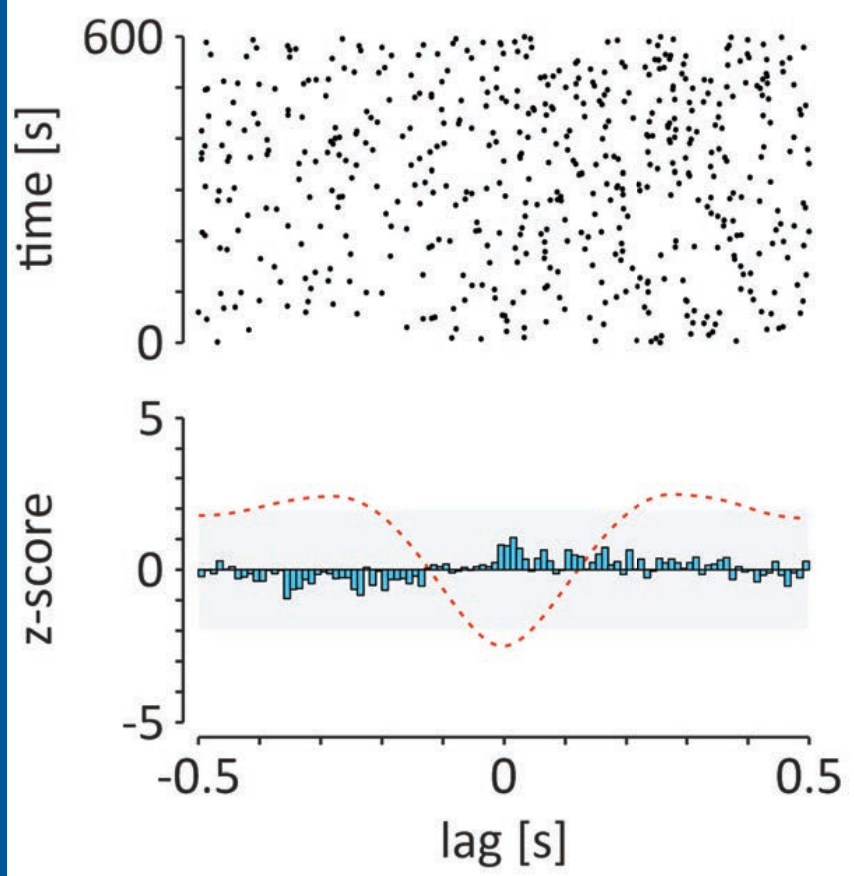

0

(a)

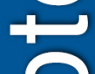

$+$

(1)

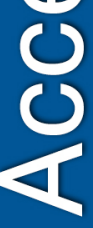

(U)

C - delta wave-independent NI neurons

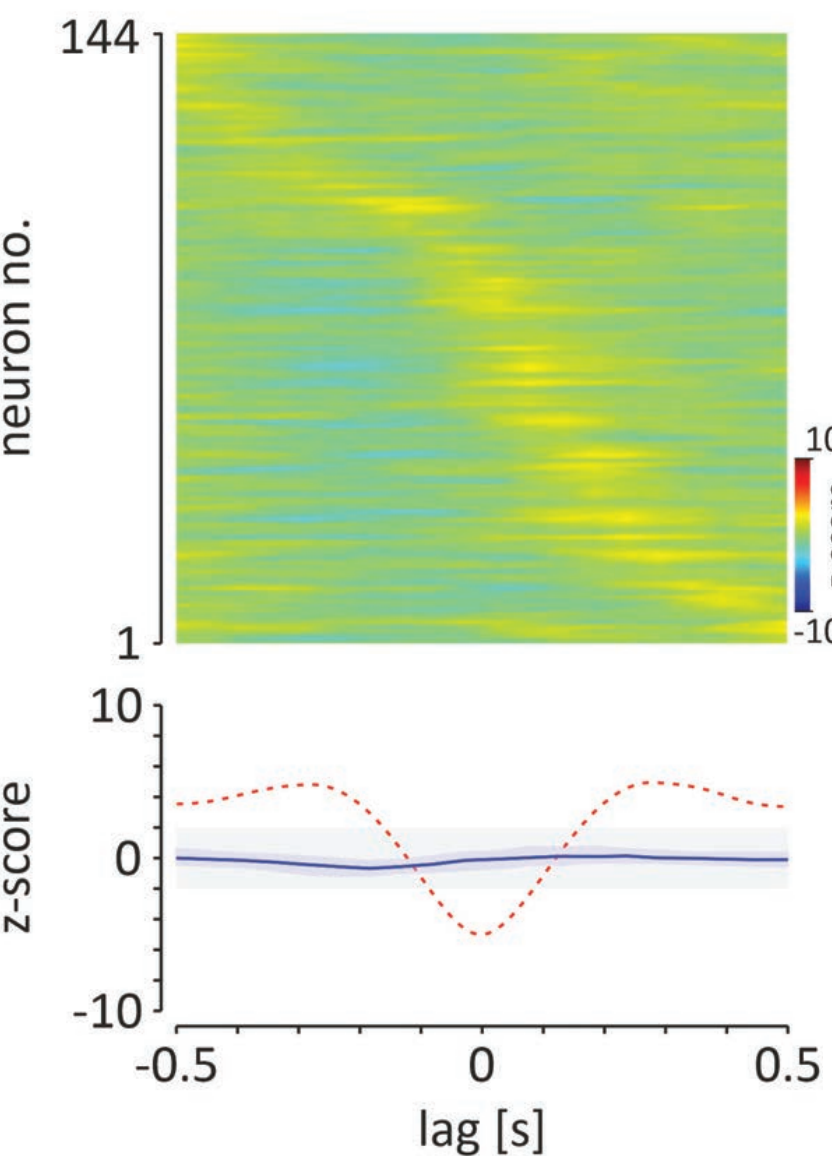

B - delta wave-locked NI neuron
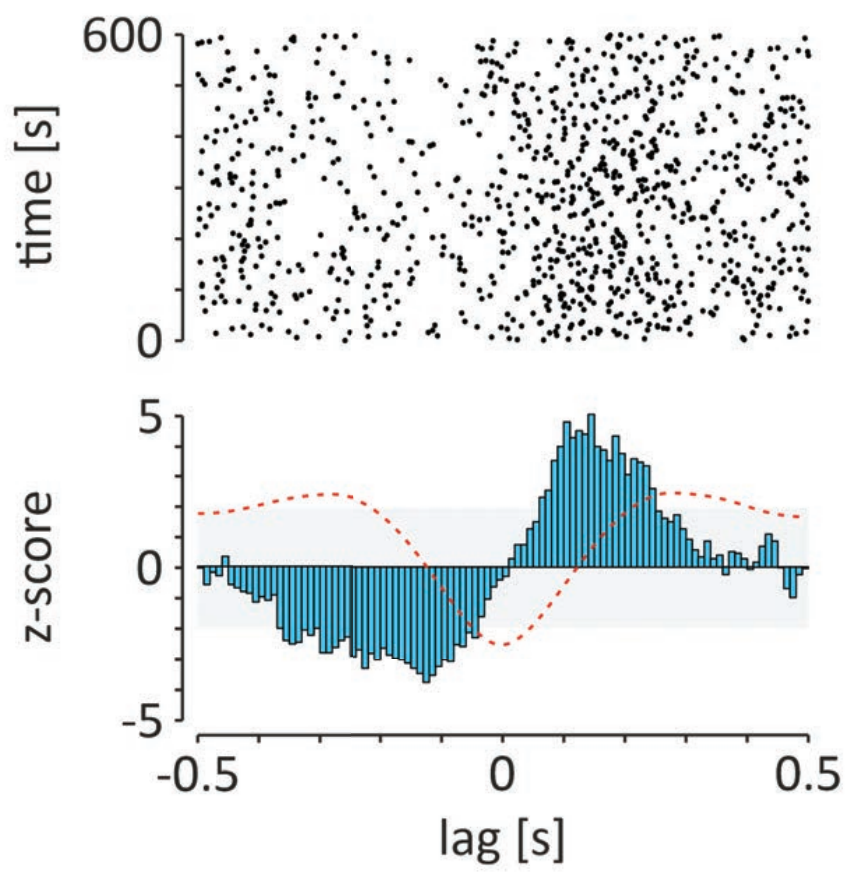

D - delta wave-locked NI neurons
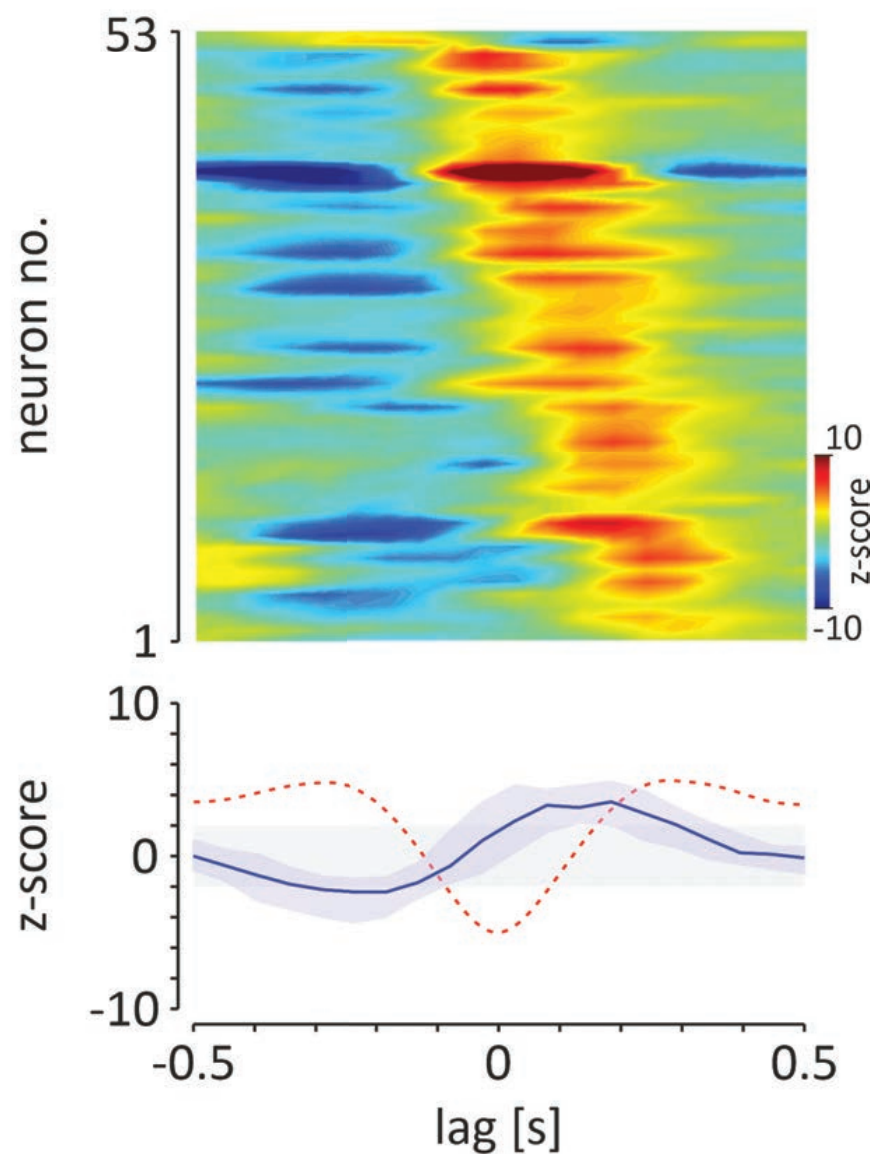


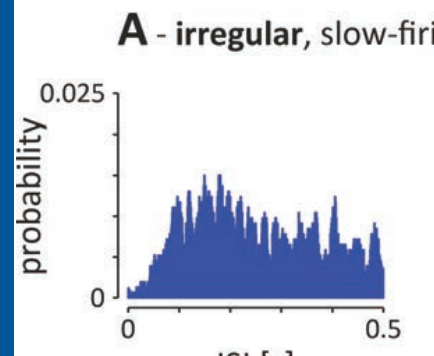

ISI [s]

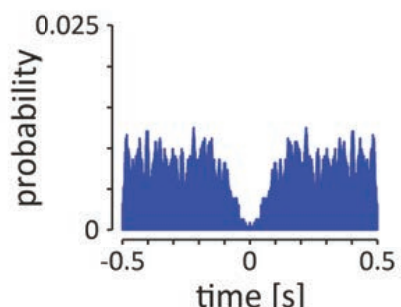

time $[s]$
C - non-bursting

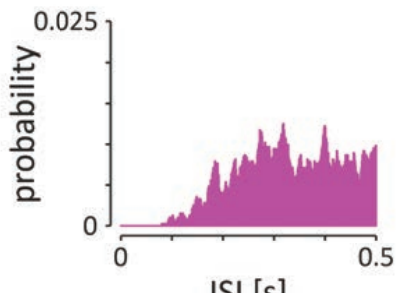

ISI [s]
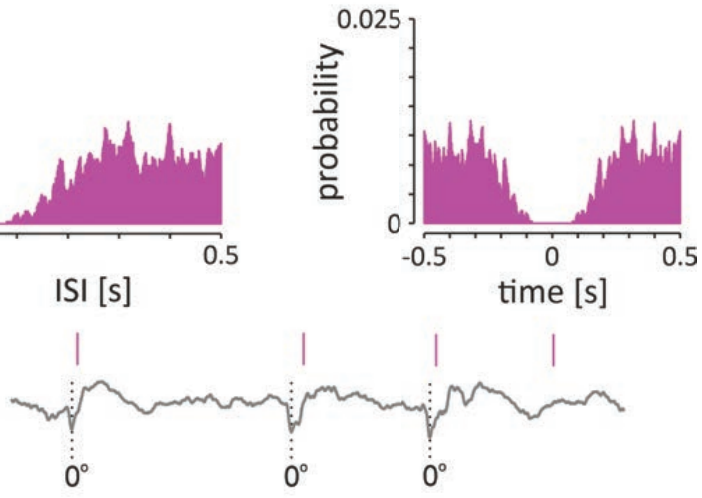

B - regular, fast-firing
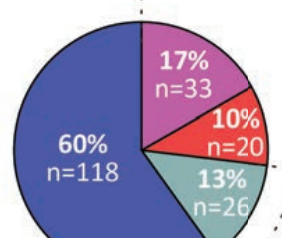

D - bursting
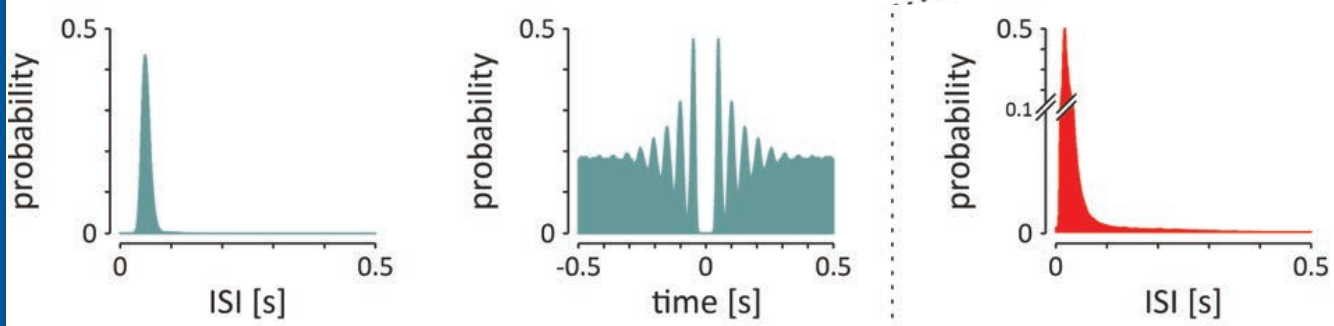

ISI [s]

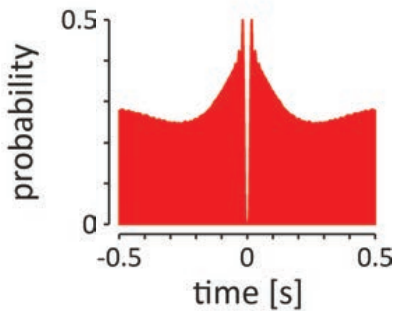

|||||||||||||||||||||||||||||||||||||||||||||||||||||||||||||||||||||||||||||||||||||||||||-

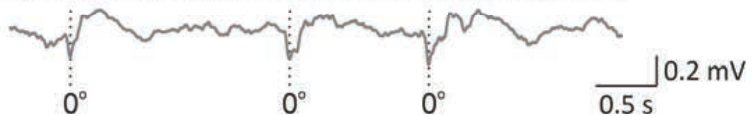

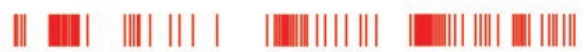

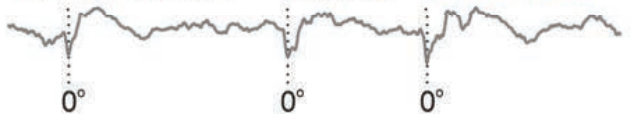


Delta wave-locked NI neurons

A D-bst. vs. D-bst.
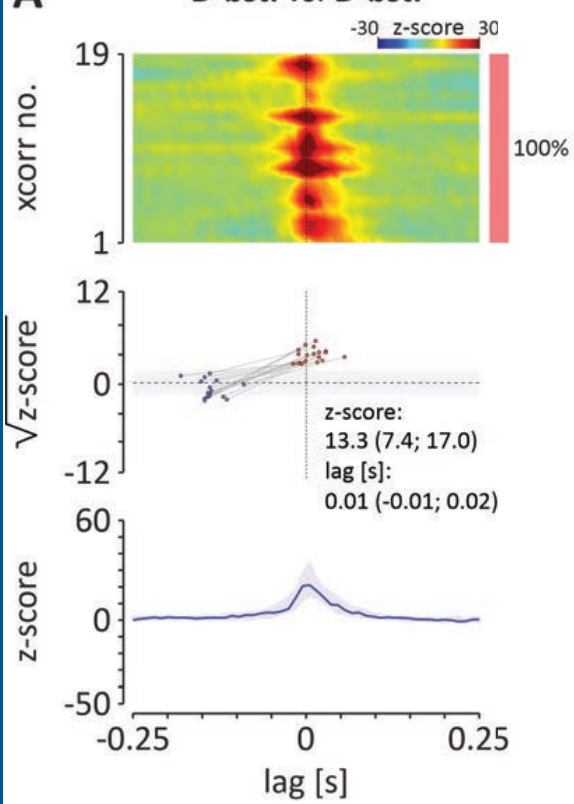

B non-bst. vs. D-bst.
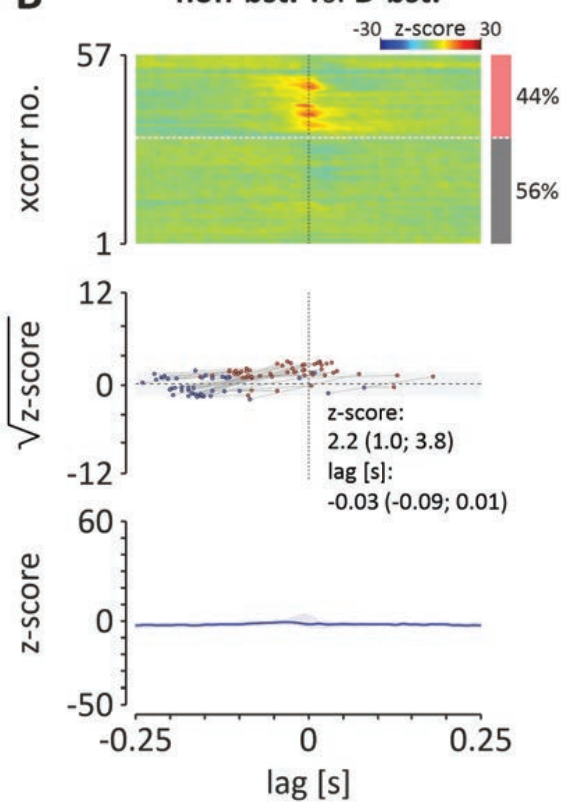

C non-bst. vs. non-bst.
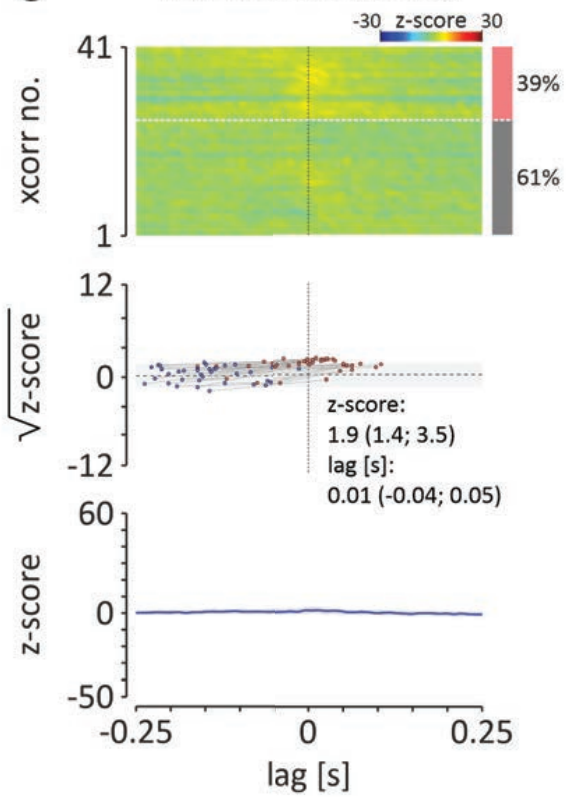

F reg. vs. reg.
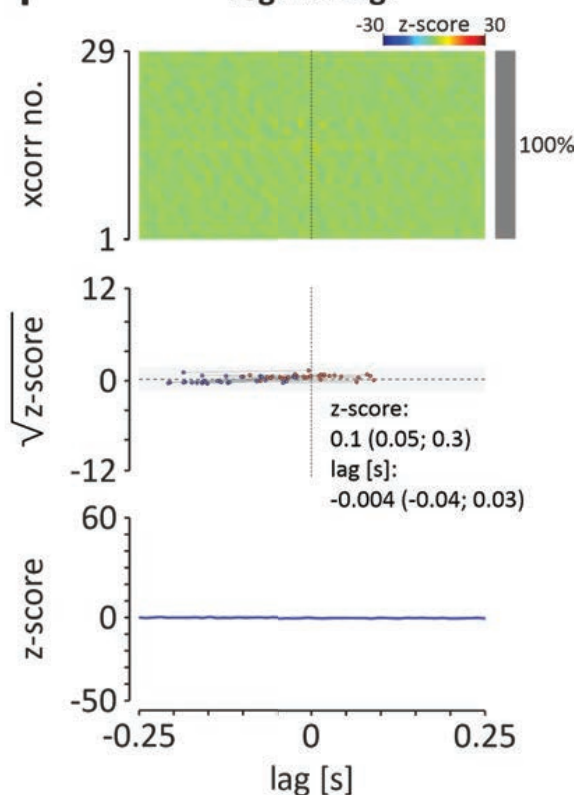
A

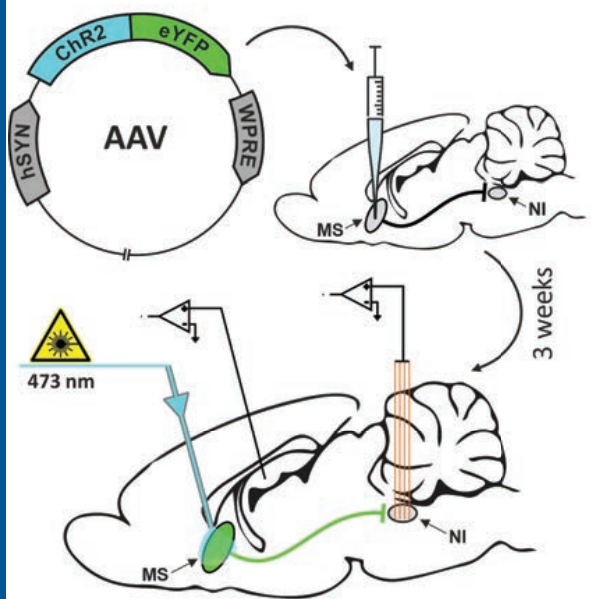

(0)

(d)

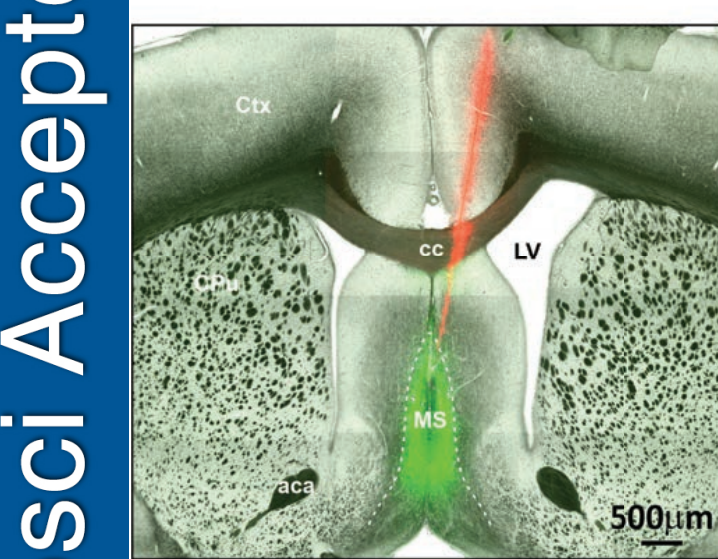

0

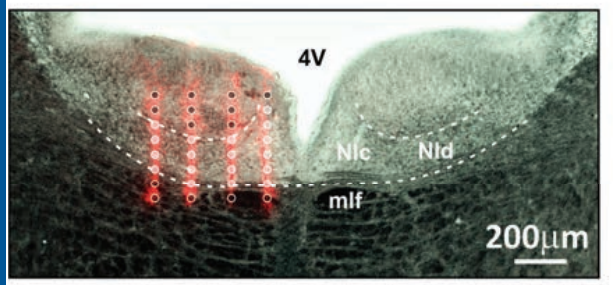

C

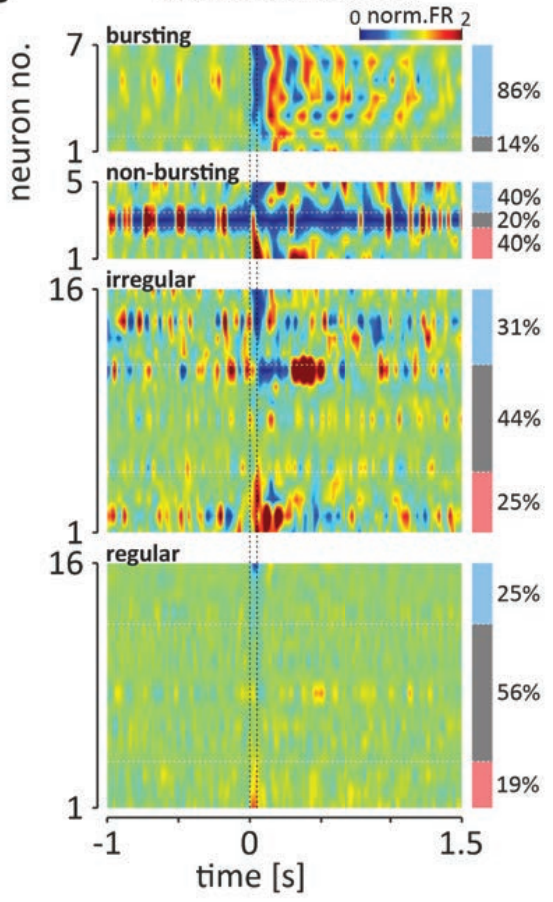

E
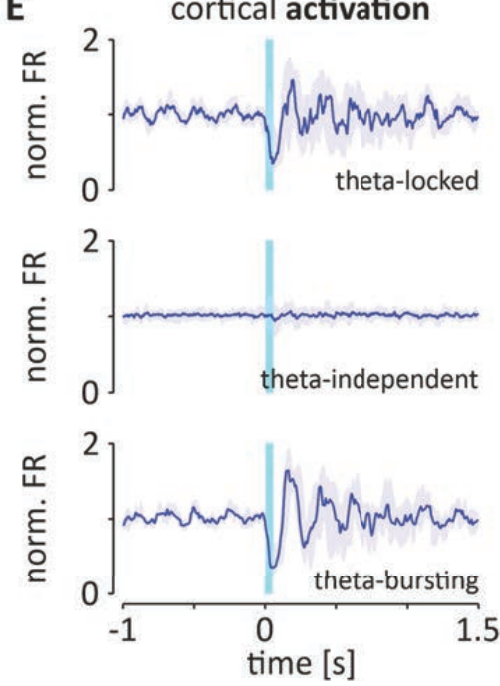

D cortical SWA

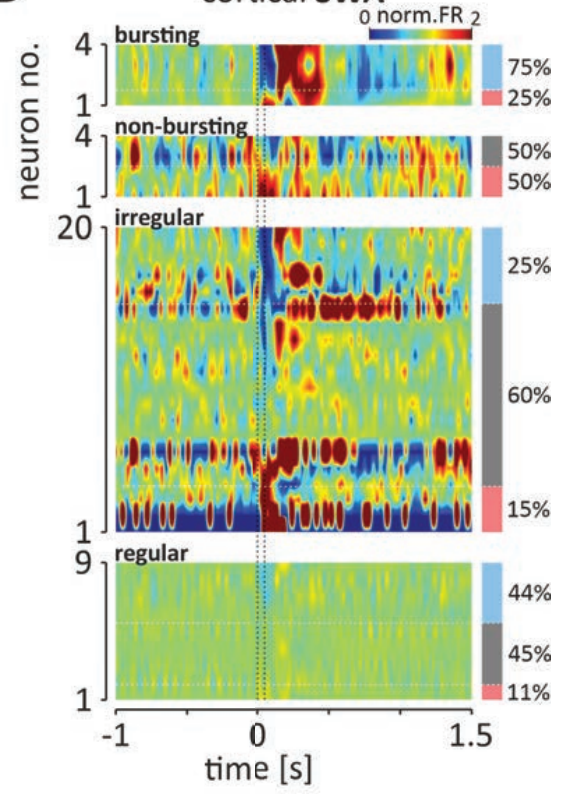

F cortical SWA
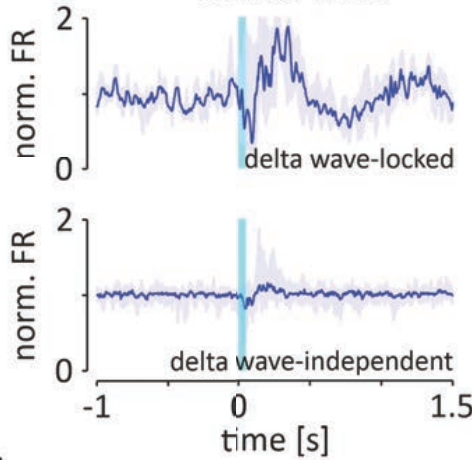

G

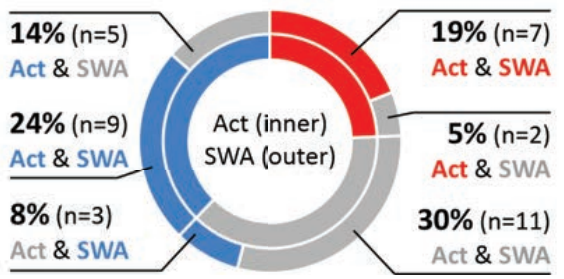




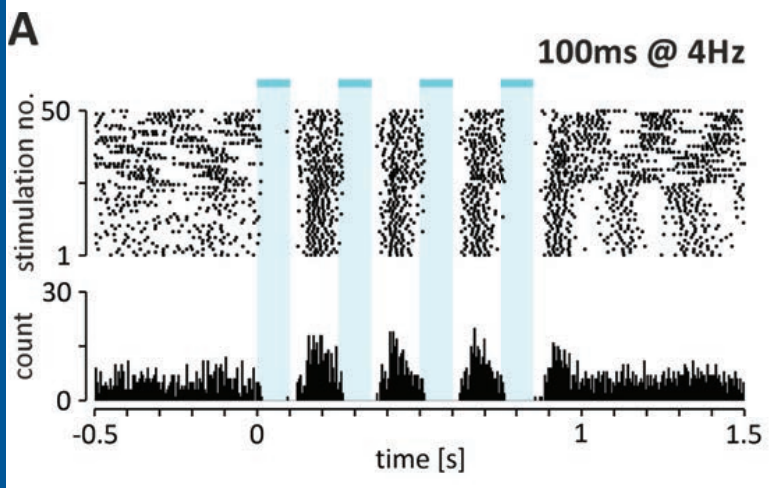

40ms@8Hz

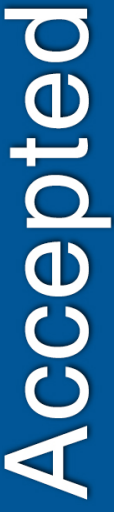

Oִ

(1)
B

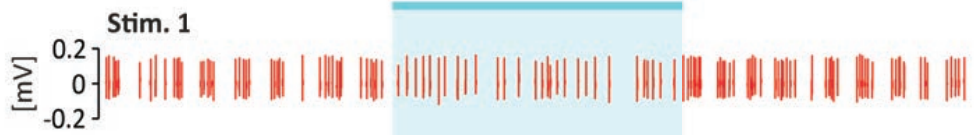

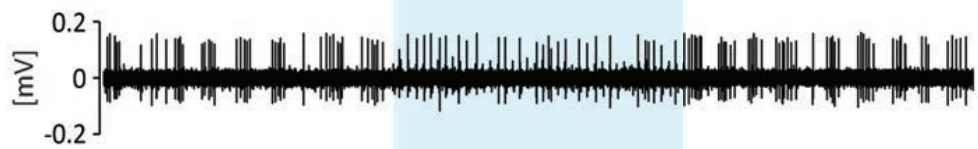

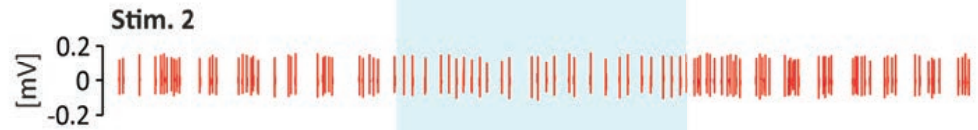

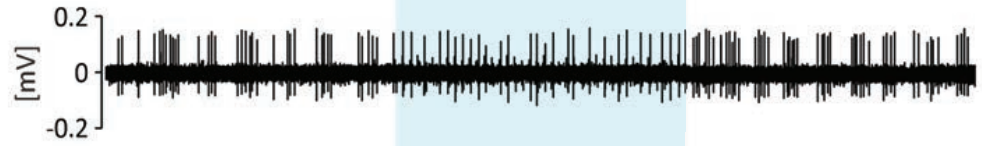

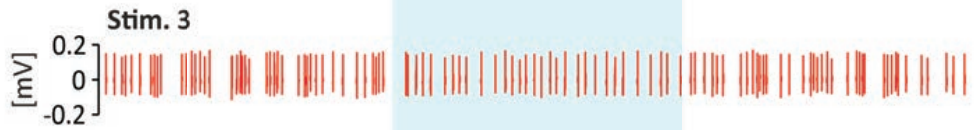

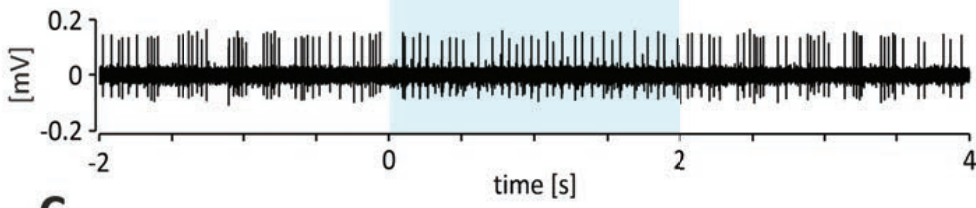
C
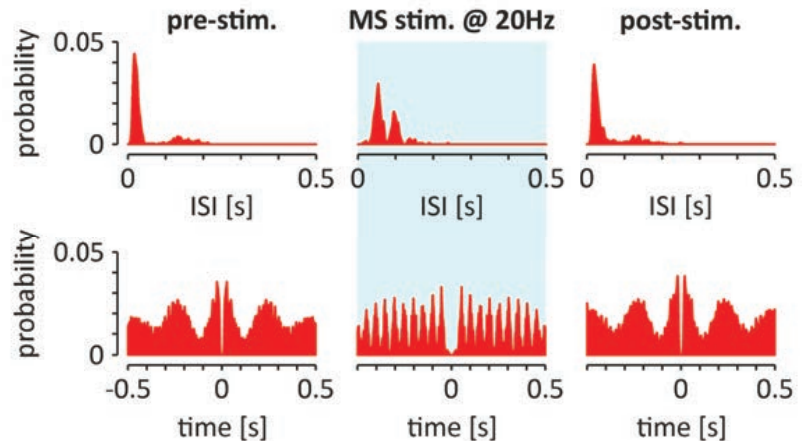

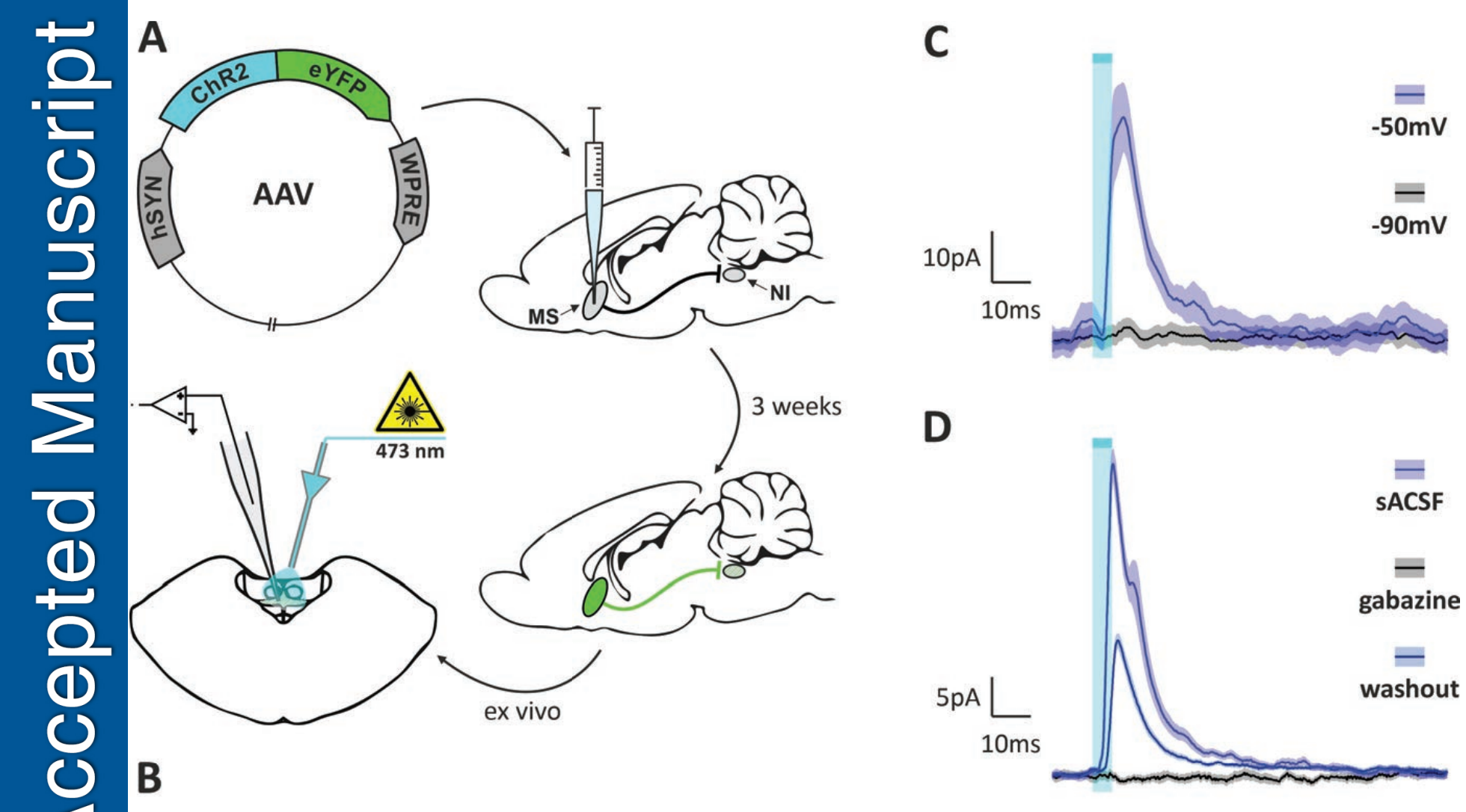

E
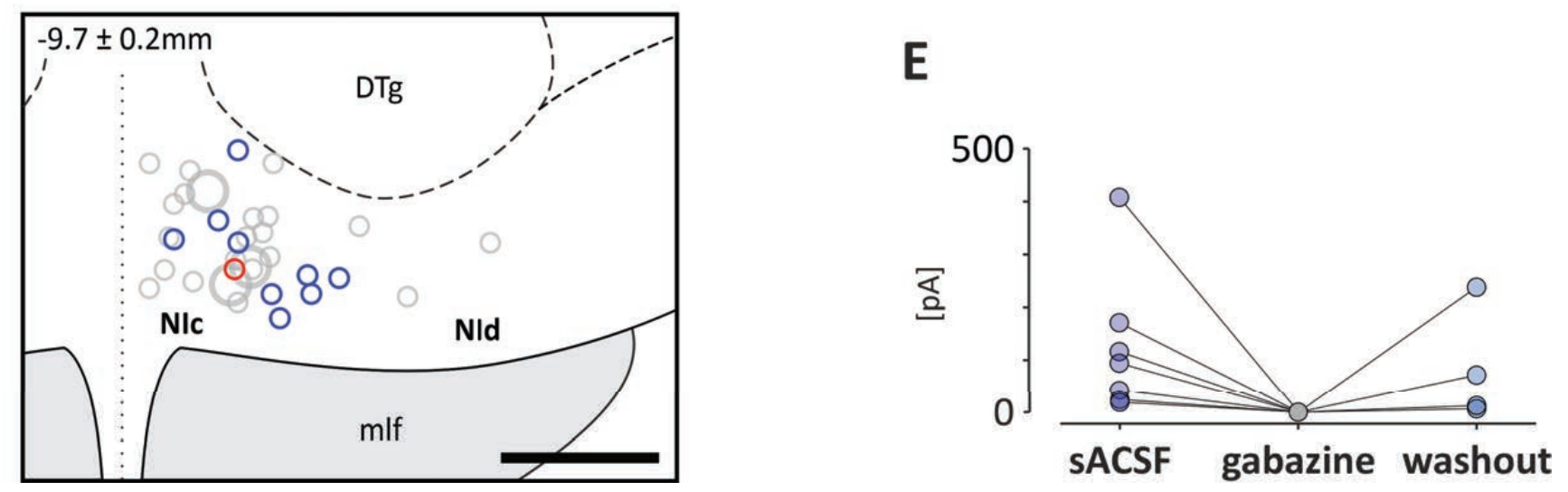

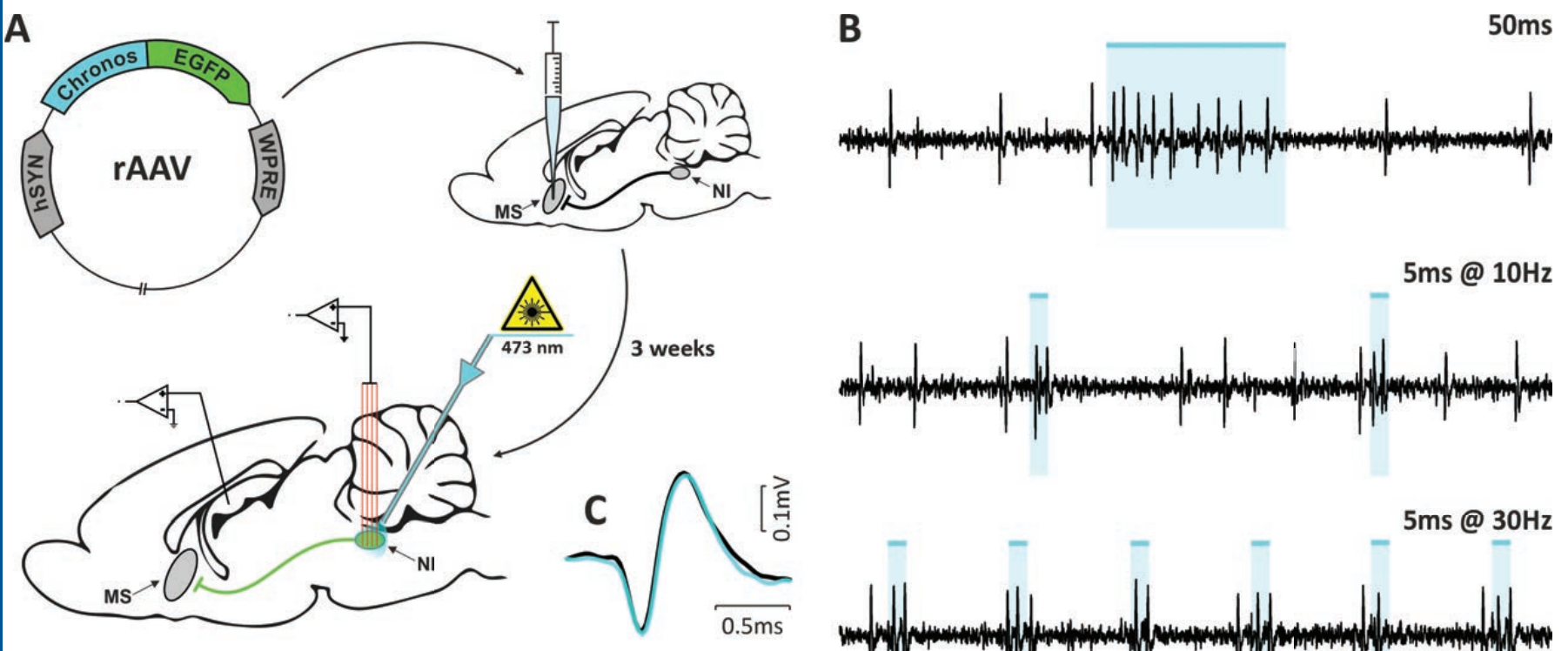

$<$

0

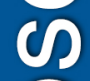

D - theta phase-independent, regular, fast-firing
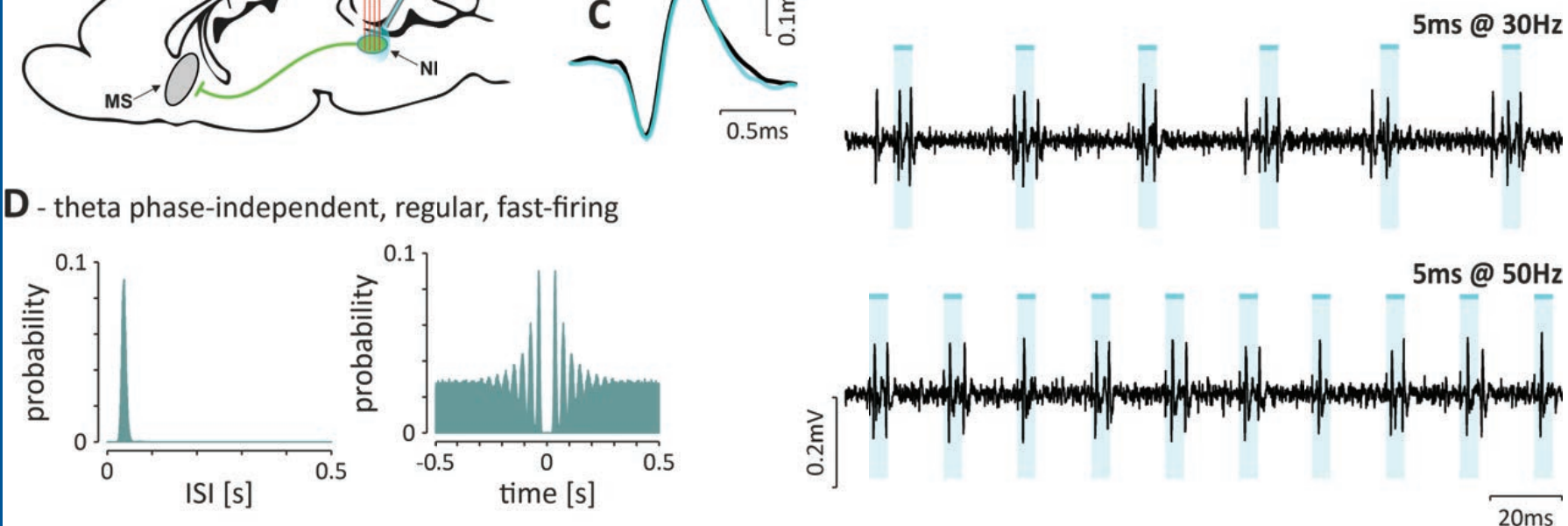

O

ISI [s]

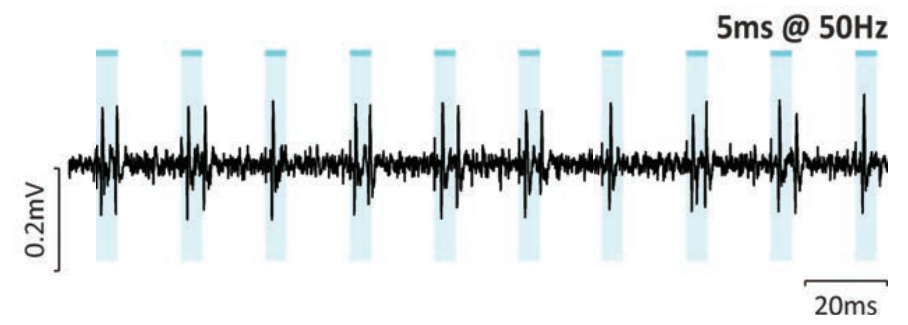




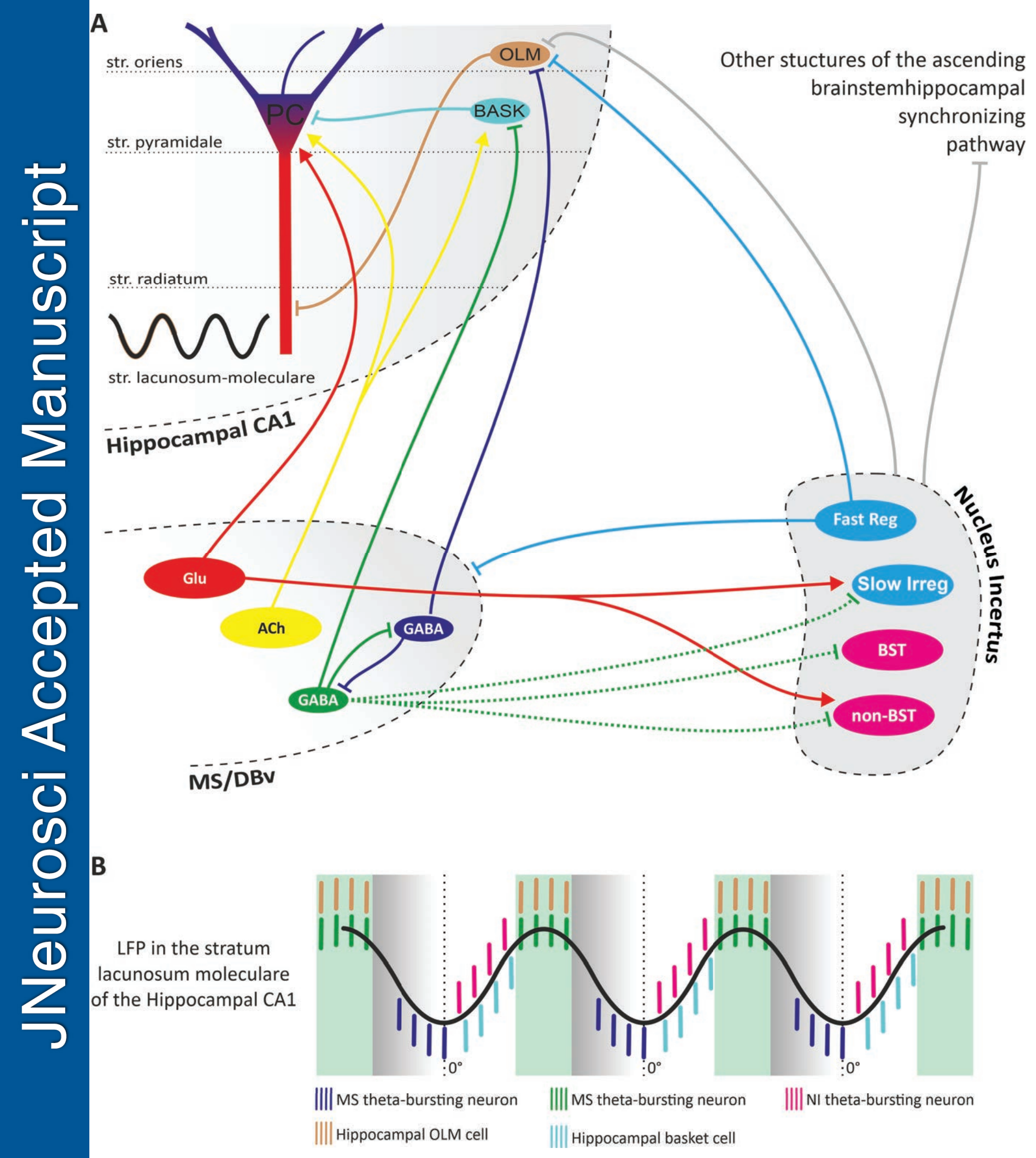

